\title{
Universality of spontaneous chiral symmetry breaking in gauge theories
}

\author{
Holger Gies* \\ CERN, Theory Division, CH-1211 Geneva 23, Switzerland
}

Christof Wetterich ${ }^{\dagger}$

Institut für theoretische Physik, Universität Heidelberg, Philosophenweg 16, D-69120 Heidelberg, Germany

(Received 30 September 2002; published 13 January 2004)

\begin{abstract}
We investigate one-flavor QCD with an additional chiral scalar field. For a large domain in the space of coupling constants, this model belongs to the same universality class as QCD, and the effects of the scalar become unobservable. This is connected to a "bound-state fixed point" of the renormalization flow for which all memory of the microscopic scalar interactions is lost. The QCD domain includes a microscopic scalar potential with minima at a nonzero field. On the other hand, for a scalar mass term $m^{2}$ below a critical value $m_{\mathrm{c}}^{2}$, the universality class is characterized by perturbative spontaneous chiral symmetry breaking which renders the quarks massive. Our renormalization group analysis shows how this universality class is continuously connected with the QCD universality class.
\end{abstract}

DOI: 10.1103/PhysRevD.69.025001

PACS number(s): 11.10.Hi, 11.30.Qc, 12.38.Aw

\section{INTRODUCTION}

The universality of QCD means that predictions are independent of the details of the microscopic interactions. This is crucial for predictivity, since the precise form of the fundamental interactions at very short distance scales is not known. In a large parameter space characterizing possible fundamental interactions, the QCD universality class corresponds, however, only to a certain domain. For other domains in parameter space, the color symmetry may be "spontaneously broken" by the Higgs mechanism, or all quarks may acquire a large mass due to spontaneous chiral symmetry breaking. We are interested here in the transition from one domain to another and in the question of what happens at the boundary of the "QCD domain."

Looking at QCD from a microscopic scale-say a unification scale $10^{15} \mathrm{GeV}$-its universality class is characterized by eight massless gluons and a certain number of massless fermions. Perturbatively, the masses are protected by the gauge symmetry and chiral symmetries. At a much smaller scale, around $1 \mathrm{GeV}$, nonperturbative effects induce masses for all physical particles. In particular, the fermions become massive owing to chiral symmetry breaking $(\chi \mathrm{SB})$. This may be described by a nonzero expectation value $\sigma \sim\langle\bar{\psi} \psi\rangle$ of a "composite" scalar field. In order to keep the discussion simple, we concentrate here on the case of one quark flavorgeneralizations to several flavors are straightforward.

Let us now consider a class of microscopic theories with a complex fundamental "chiral scalar field" $\phi$ which has the same transformation properties as $\bar{\psi} \psi$ and a classical potential

$$
V=m^{2} \phi^{*} \phi+\frac{1}{2} \lambda_{\phi}\left(\phi^{*} \phi\right)^{2} .
$$

\footnotetext{
*Email: Holger.Gies@cern.ch

${ }^{\dagger}$ Email: C.Wetterich@thphys.uni-heidelberg.de
}

The symmetries also allow for a Yukawa coupling between $\phi$ and the quarks. For nonzero $\langle\phi\rangle$, the chiral symmetry is broken and the quarks become massive. In the case of large enough positive $\mathrm{m}^{2}$ (in units of some unification scale, say $10^{15} \mathrm{GeV}$ ), the scalar field is super-heavy and decouples from the low-energy theory. This range of $\mathrm{m}^{2}$ obviously corresponds to the universality class of QCD. All effects of the scalar field are suppressed by $p^{2} / \mathrm{m}^{2}$, with $p$ a characteristic momentum. For QCD predictions, they can be completely ignored.

On the other hand, for large enough negative $m^{2}$, we expect the perturbative picture of spontaneous symmetry breaking to hold. The scalar field gets a vacuum expectation value (VEV)

$$
\langle\phi\rangle=\sigma=\left|m_{\mathrm{R}}^{2} / \lambda_{\phi, \mathrm{R}}\right|^{1 / 2}
$$

with $m_{\mathrm{R}}$ and $\lambda_{\phi, \mathrm{R}}$ related to $m$ and $\lambda_{\phi}$ by renormalization corrections. Both $\sigma$ and the quark masses are of the order of the unification scale in this domain. The universality class now corresponds to gluodynamics without light quarks. In the chiral limit of a vanishing current quark mass, spontaneous $\chi \mathrm{SB}$ also generates a very light pseudo-Goldstone boson in addition to the gluonic degrees of freedom.

Varying the microscopic scalar mass term $\mathrm{m}^{2}$ from large negative to large positive values should lead us from the universality class with perturbative spontaneous chiral symmetry breaking $(\mathrm{P} \chi \mathrm{SB})$ to the universality class of one-flavor QCD. One of the aims of this note is to understand the qualitative features of this transition in the vicinity of a critical value $m_{\mathrm{c}}^{2}$. This is clearly a nonperturbative problem, since on the QCD side of the transition the effective gauge coupling grows large.

Our investigation is based on a nonperturbative flow equation which is obtained by a truncation of the exact renormalization group equation for the effective average action [1]. A crucial ingredient is the "bosonization" of effective multi-fermion interactions at every scale [2]. This provides for a description of fundamental scalar fields and bound 
states in a unified framework. A theoretical method with this feature is actually required for our problem, since the scalar quark-antiquark bound states in the QCD description (e.g., the pseudo-Goldstone eta meson and the sigma meson) are expected to become associated with the fundamental scalar in the $\mathrm{P} \chi \mathrm{SB}$ description. In this framework, we see also how one relevant parameter and two marginal parameters in the $\mathrm{P} \chi \mathrm{SB}$ universality class, namely, the ones corresponding to the mass and quartic self-interaction of the scalar field and the Yukawa coupling, become irrelevant for the QCD universality class.

This remarkable change of the number of relevant parameters at the transition between the two universality classes is connected with the appearance of a bound-state fixed point for the flow of the scalar mass and self-interaction in the range of microscopic parameters corresponding to QCD. This bound-state fixed point is infrared attractive for all couplings except for the gauge coupling. Under the influence of this fixed point, all memory of the details of the microscopic interactions in the scalar sector is lost. This is exactly what is required for the QCD universality class which has the gauge coupling as the only marginal parameter (for a massless quark). In order to see the appearance of the bound state, it is crucial to reincorporate the effective multi-fermion interactions generated by the flow into the effective bosonic interactions. This avoids an unwanted redundancy of the description. It also solves an old problem in the investigation of gauged Nambu-Jona-Lasinio models [3]; namely, how the presence of apparent relevant parameters in a too naive treatment of these models can be reconciled with QCD, where no such relevant parameters are present. In our approach, the flow towards the bound-state fixed point solves this generic problem.

As a result of our investigation, we find a qualitatively convincing picture of the transition between the two universality classes investigated. We have kept the truncation simple in order to illustrate the change in the number of relevant and marginal parameters in a simple way. The price to be paid is a limited accuracy in the quantitative description for parameter regions where the effective gauge coupling grows large. In our setting, this concerns primarily the quantitative details of the flow of the instanton-mediated interactions and the running of the strong gauge coupling. We emphasize that the qualitative picture does not require a detailed understanding of strong interactions in the momentum range where the gauge coupling is large. All decisive features are determined by the flow in a momentum range substantially larger than $1 \mathrm{GeV}$. In the same spirit, we also have neglected other effective bosonic degrees of freedom which may correspond to additional bound states. We keep only the composite scalars and the gluons. When we proceed with our analysis to the strongly coupled gauge sector, we do not attempt to compute the gluodynamics, but simply model the strong interactions with an increasing gauge coupling; for the latter, we use various examples discussed in Appendix B. We do not claim that our truncation of the gauge sector is sufficient in order to establish chiral symmetry breaking in QCD. A much more elaborate analysis would be needed for this purpose. We rather take the spontaneous symmetry breaking in the QCD universality class as a fact (established by other methods and observation). We only require that a reasonable truncation should describe chiral symmetry breaking. Beyond this, the details of the truncation in the gauge sector are not relevant for our discussion of universality classes. Despite these shortcomings, we expect that our quantitative results describe the right order of magnitude of one-flavor QCD. An impression of the size of uncertainties can be gained from Table I in Appendix B.

In order to illustrate our points, we compute the scalar condensate, i.e., the renormalized minimum of the effective potential, $\sigma_{R}=\sqrt{Z_{\phi}\left|\phi_{0}\right|^{2}}$, for a broad range of initial scalar mass values $\bar{m}_{\Lambda}^{2}$. We note that $\sigma_{\mathrm{R}}$ is directly connected with the decay constant of the eta meson and sets the scale for the quark mass generated by $\chi \mathrm{SB}$. We first neglect the anomalous $\mathrm{U}_{\mathrm{A}}(1)$ violating contributions from instanton effects which only affect the physics at scales around $1 \mathrm{GeV}$. (They will be considered in Sec. V.) We parametrize the microscopic interactions by the initial values of the renormalization flow at a GUT-like scale $\Lambda=10^{15} \mathrm{GeV}$. As can be read from Fig. 1, a critical mass $\bar{m}_{\mathrm{c}}^{2}$ exists. For initial scalar masses below this critical mass, $\bar{m}_{\Lambda}^{2}<\bar{m}_{\mathrm{c}}^{2}$, the naive expectation is fulfilled, and we find scalar condensates of the order of the cutoff, $\sigma_{R} \sim 10^{13} \ldots 10^{15} \mathrm{GeV}$. It is remarkable that the value of the critical mass is negative and typically of the order of the cutoff or only a few orders of magnitude below the cutoff; for example, we find $\bar{m}_{\mathrm{c}}^{2} \simeq-0.35 \Lambda^{2}$ for the initial values $\bar{h}^{2}=1$ and $\bar{\lambda}_{\phi}=100$ at $\Lambda=10^{15}$ [Fig. 1 (left panel)]. For a perturbatively accessible set of initial parameters $\bar{h}^{2}$ $=0.1$ and $\bar{\lambda}_{\phi}=1$ at $\Lambda=10^{15}$, we find $\bar{m}_{\mathrm{c}}^{2} \simeq-0.0043 \Lambda^{2}$ [Fig. 1 (right panel)]. In the latter case, we find a linear dependence of the condensate on the mass parameter, $\sigma_{\mathrm{R}}^{2}$ $\sim-\left(\bar{m}_{\Lambda}^{2}-\bar{m}_{\mathrm{c}}^{2}\right)$, as expected from perturbation theory [cf. Eq. (2)].

However, for initial scalar masses above this critical mass, $\bar{m}_{\Lambda}^{2}>\bar{m}_{\mathrm{c}}^{2}$, the scalar condensate is 16 orders of magnitude smaller [not visible in the linear plot in Fig. 1 (right panel)]. In this case, symmetry breaking is triggered by the fermion and gauge sectors and not by the scalar sector, i.e., $\sigma_{R}$ is roughly of the order of $\Lambda_{\mathrm{QCD}}$. Therefore, even if we start the flow deep in the broken regime with $\bar{m}_{\Lambda}^{2}<0$ but above the critical mass, the scalar fluctuations drive the system first into the symmetric regime where it will be attracted by the same IR fixed point as a QCD-like system. It should be stressed that no fine tuning of the initial parameters is needed, neither to put the system into the domain of attraction of the QCD universality class nor to separate the ultraviolet (UV) scale from the scale of chiral symmetry breaking.

Only for $\bar{m}_{\Lambda}^{2}<\bar{m}_{\mathrm{c}}^{2}$ is the effective coupling between the scalars and the fermions strong enough to induce $\mathrm{P} \chi \mathrm{SB}$ with a magnitude determined by the initial parameters of the scalar sector. In this case, we would have to fine-tune the initial condition for $\bar{m}_{\Lambda}^{2}$ to lie extremely close to $\bar{m}_{\mathrm{c}}^{2}$, if we wanted to separate the UV scale from the scale of chiral symmetry breaking. This is the famous naturalness problem which is 

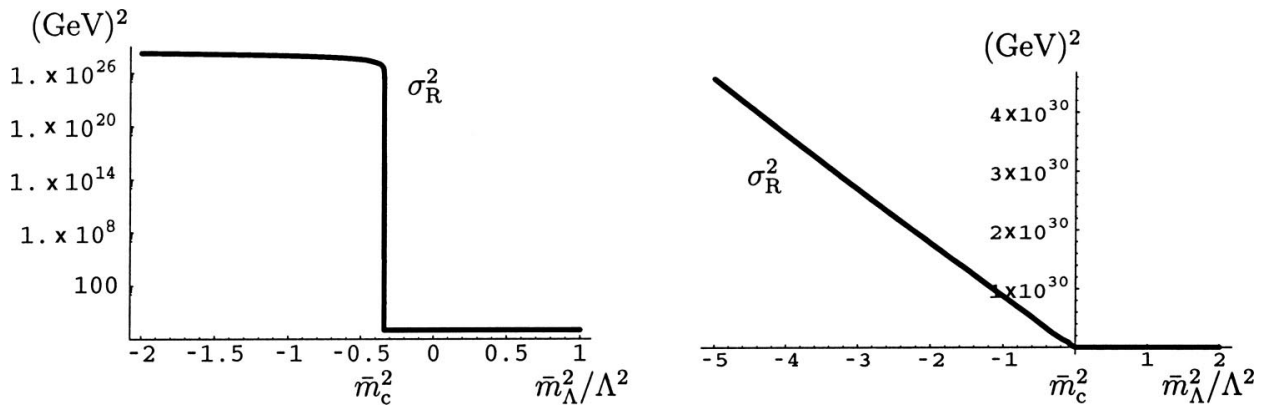

FIG. 1. Renormalized scalar vacuum expectation value $\sigma_{\mathrm{R}}^{2}$ versus the initial condition for the scalar mass at the UV cutoff, $\bar{m}_{\Lambda}^{2}$. Left panel: logarithmic plot for the parameters $Z_{\phi}=1, \bar{h}^{2}=1, \bar{\lambda}_{\phi}=100$ at $\Lambda=10^{15} \mathrm{GeV}$ resulting in $\bar{m}_{\mathrm{c}}^{2} \simeq-0.35 \Lambda^{2}$. Right panel: linear plot for $\bar{h}^{2}=0.1, \bar{\lambda}_{\phi}=1$; for $\bar{m}_{\Lambda}^{2}<\bar{m}_{\mathrm{c}}^{2} \simeq-0.0043 \Lambda^{2}$, the linear dependence $\sigma_{\mathrm{R}}^{2} \sim-\left(\bar{m}_{\Lambda}^{2}-\bar{m}_{\mathrm{c}}^{2}\right)$, as expected from perturbation theory, is confirmed.

generic for models involving a fundamental scalar. Of course, theories without fundamental scalars such as QCD do not have this problem, although effective scalar degrees of freedom such as bound states can occur at low energies. It is one of our main observations that the mechanism of how "QCD-like" theories circumvent the naturalness problem can also be applied to models with a fundamental scalar.

The details of our study are organized as follows: in Sec. II, we introduce the class of models containing one-flavor QCD and derive the flow equations for a qualitatively reliable truncation including "bosonization at all scales." Section III is devoted to a discussion of the bound-state fixed point which governs the flow of the QCD domain for weak gauge coupling. In Sec. IV, we analyze the universal features of the QCD domain numerically and give estimates of infrared (IR) observables in the nonperturbative strong-coupling regime. Instanton-mediated interactions are included in Sec. $\mathrm{V}$, where we also describe the fate of the pseudo-Goldstone boson.

\section{FLOW EQUATIONS}

QCD with one massless Dirac fermion flavor coupled to an $\mathrm{SU}\left(N_{\mathrm{c}}\right)$ gauge field is characterized by the classical (or bare) action

$$
S_{\mathrm{QCD}}=\int d^{4} x \bar{\psi} \mathrm{i} \not D[A] \psi+\frac{1}{4} F_{\mu \nu}^{a} F_{\mu \nu}^{a},
$$

where $D_{\mu}^{i j}[A]=\partial_{\mu} \delta^{i j}-\mathrm{i} \bar{g} T_{a}^{i j} A_{\mu}^{a}$, and $T_{a}$ denotes the (Hermitian) generators of the gauge group in the fundamental representation. In this work, we embed one-flavor QCD in a larger class of chirally invariant theories including a colorsinglet scalar field. For this, we consider the action

$$
\begin{aligned}
\Gamma= & \int\left\{Z_{\psi} \bar{\psi} \mathrm{i} \not D \psi+\frac{\bar{\lambda}_{\sigma}}{2}\left[(\bar{\psi} \psi)^{2}-\left(\bar{\psi} \gamma_{5} \psi\right)^{2}\right]+Z_{\phi} \partial_{\mu} \phi^{*} \partial_{\mu} \phi\right. \\
& +U(\phi)+\bar{h}\left[\left(\bar{\psi}_{\mathrm{R}} \psi_{\mathrm{L}}\right) \phi-\left(\bar{\psi}_{\mathrm{L}} \psi_{\mathrm{R}}\right) \phi^{*}\right] \\
& \left.+\frac{Z_{\mathrm{F}}}{4} F_{\mu \nu}^{a} F_{\mu \nu}^{a}+\frac{1}{2 \xi}\left(\bar{D}_{\mu} a_{\mu}^{a}\right)^{2}\right\},
\end{aligned}
$$

which represents a simple truncation of the space of action functionals and serves as the basis of our approximations. Here we have used the shorthand $(\bar{\psi} \psi)=\bar{\psi}^{i} \psi_{i}$ for the color indices. We included a background gauge fixing term with parameter $\xi$, and $A_{\mu}=\bar{A}_{\mu}+a_{\mu}, \bar{A}_{\mu}$ being the background and $a_{\mu}$ the fluctuation field, $\bar{D}_{\mu} \equiv D_{\mu}[\bar{A}]$. Furthermore, we do not display the ghost sector for simplicity. Equation (4) reduces to one-flavor QCD if we set the four-fermion and the Yukawa interaction equal to zero, $\bar{\lambda}_{\sigma}=\bar{h}=0$, let the scalar field be auxiliary, $Z_{\phi}=0$, and set $Z_{F}=1=Z_{\psi}$ (the scalar potential is of no importance then). Furthermore, there is a redundancy in Eq. (4): we can compensate for a shift in $\bar{\lambda}_{\sigma}$ by readjusting the Yukawa coupling and the scalar potential corresponding to a Hubbard-Stratonovich transformation (partial bosonization). But apart from this redundancy, which will be removed later on by "rebosonization," different initial values for the various parameters in Eq. (4) generally correspond to different quantum theories. Some of these theories will belong to the same universality class sharing the same low-energy properties, which makes them indistinguishable from a low-energy physicist's point of view.

We analyze this class of theories in a Wilsonian spirit upon integrating out quantum fluctuations momentum shell by momentum shell. For this we employ the formalism based on the exact renormalization group flow equation for the effective average action $[1,4]$,

$$
\partial_{t} \Gamma_{k}=\frac{1}{2} \operatorname{STr}\left[\partial_{t} R_{k}\left(\Gamma_{k}^{(2)}+R_{k}\right)^{-1}\right]
$$

where $\Gamma_{k}^{(2)}$ denotes the second functional derivative of the effective average action $\Gamma_{k}$ that governs the dynamics of the system at a momentum scale $k$. The logarithmic scale parameter $t$ is given by $t=\ln k / \Lambda, \partial_{t}=k(d / d k)$, where $\Lambda$ denotes the ultraviolet scale at which we define the bare action $\Gamma_{\Lambda}$. The cutoff function $R_{k}$ is to some extent arbitrary and obeys a few restrictions [4] which ensure that the flow is well defined and interpolates between the bare action in the UV and the full quantum effective action $\Gamma_{k \rightarrow 0}$ in the infrared.

We solve the flow equation (5) by using Eq. (4) as a truncation of the space of all possible action functionals. As a consequence, we promote all couplings and wave function 
renormalizations occurring in Eq. (4) to $k$-dependent quantities. Although the truncation (4) represents only a small subclass of possible operators generated by quantum fluctuations, it is able to capture many physical features of QCDlike systems.

Let us elucidate the single components in detail: for the scalar potential, we use the simple truncation

$$
U(\phi)=\bar{m}^{2} \rho+\frac{1}{2} \bar{\lambda}_{\phi} \rho^{2}-\frac{1}{2} \bar{\nu} \zeta, \quad \rho=\phi^{*} \phi, \quad \zeta=\phi+\phi^{*} .
$$

Already the $\rho$-dependent first two terms of the potential are capable of describing spontaneous $\chi \mathrm{SB}$ of the system which we are aiming at. Indeed, the order parameter $\sigma$ denotes the minimum of the scale-dependent effective potential $U_{k}$ for $k \rightarrow 0$. The term $\sim \zeta=\phi+\phi^{*}$ breaks the $\mathrm{U}_{\mathrm{A}}(1)$ symmetry of simultaneous axial phase rotations of scalars and fermions; it accounts for the effects of the axial anomaly. However, the presence of the axial anomaly is not relevant for universality of spontaneous $\chi \mathrm{SB}$, although it has, of course, a strong quantitative impact on resulting low-energy parameters such as condensates and constituent quark masses. Therefore, we postpone the discussion of this quantitative influence to Sec. $\mathrm{V}$ and set $\bar{\nu}=0$ in the following for the sake of clarity.

In the gauge sector, we do not attempt to calculate the full nonperturbative flow of $Z_{\mathrm{F}}$, or alternatively the gauge coupling $g$, here, but study various possibilities for these flows and take over nonperturbative results from the literature. The most important features of the universality classes involve only the perturbative running of $g .{ }^{1}$

We will define the quantum theories by fixing the initial conditions for the renormalization flow at the UV scale $\Lambda$. In the gauge and fermion sectors, we choose

$$
\left.Z_{\mathrm{F}}\right|_{k=\Lambda}=1,\left.\quad Z_{\psi}\right|_{k=\Lambda}=1,\left.\quad \bar{\lambda}_{\sigma}\right|_{k=\Lambda}=0 .
$$

The first two conditions normalize the gauge and fermion fields and imply that $\bar{g}$ denotes the bare gauge coupling. The last condition states that four-fermion interactions either have been partially bosonized into the scalar sector or are completely absent at the UV cutoff scale $\Lambda$.

The choice of the scalar couplings at the UV cutoff will finally determine whether we are in or beyond the QCD domain. In order to describe standard QCD in our picture, a natural choice is given by

$$
\left.\bar{m}^{2}\right|_{k=\Lambda}=+\mathcal{O}\left(\Lambda^{2}\right),\left.\quad \bar{\lambda}_{\phi}\right|_{k=\Lambda}=0,\left.\quad\left(Z_{\phi}, \bar{h}\right)\right|_{k=\Lambda} \rightarrow 0,
$$

implying that the scalar fields are nondynamic, noninteracting and heavy at $\Lambda$ and decouple from the fermion sector.

\footnotetext{
${ }^{1}$ The running of $g$ is universal in two-loop order. In the framework of the exact renormalization group, this has been computed in Ref. [8]. As discussed in the introduction, the one-loop running is actually sufficient to generate the main qualitative features needed for our argument.
}

They could be integrated out without any effect on the fermion sector and therefore are completely auxiliary. However, we will demonstrate below that the infrared physics including $\chi \mathrm{SB}$ is to a large extent independent of the initial values in the scalar sector; in other words, the QCD universality class is actually much bigger than the restrictive choice of initial conditions of Eq. (8). ${ }^{2}$

For a concise presentation of the renormalization group (RG) flow equations of the single couplings, it is convenient to introduce the dimensionless, renormalized and $k$ dependent quantities,

$$
\epsilon=\frac{\bar{m}^{2}}{Z_{\phi} k^{2}}, \quad \lambda_{\phi}=\frac{\bar{\lambda}_{\phi}}{Z_{\phi}^{2}}, \quad h=\frac{\bar{h}}{Z_{\phi}^{1 / 2} Z_{\psi}},
$$

in the symmetric regime of the system. In the $\chi \mathrm{SB}$ regime, the mass term becomes negative, and we replace this coupling by the minimum of the potential $\rho_{0}$ and its corresponding dimensionless variable $\kappa$ defined by

$$
0=\frac{\partial}{\partial \rho} U_{k}\left(\rho=\rho_{0}\right), \quad \kappa=\frac{Z_{\phi} \rho_{0}}{k^{2}} .
$$

Similarly, we define $\bar{\lambda}_{\phi}$ as the second $\rho$-derivative of the potential at the minimum in the $\chi \mathrm{SB}$ regime. The running of the wave function renormalizations is studied using the associated anomalous dimensions,

$$
\eta_{\phi}=-\partial_{t} \ln Z_{\phi}, \quad \eta_{\psi}=-\partial_{t} \ln Z_{\psi}, \quad \eta_{\mathrm{F}}=-\partial_{t} \ln Z_{\mathrm{F}}
$$

where $\eta_{\mathrm{F}}$ represents the major piece of information from the gauge sector in our truncation. Here, the use of the background-field method for this gauge sector has two advantages: first, it represents a bookkeeping device to set up consistent gauge-invariant approximations within a certain order of truncation. Second, the physical idea of the background field is that it accommodates the true ground state of the system around which the quantum fluctuations are integrated out. In this spirit, we deduce the running gauge coupling from the RG behavior of the background field. Owing to background gauge invariance, the product of gauge coupling and background gauge field is renormalization-group invariant [5], so that the beta function for the renormalized running gauge coupling $g$ is related to $\eta_{\mathrm{F}}$ by

$$
\beta_{g^{2}} \equiv \partial_{t} g^{2}=\eta_{\mathrm{F}} g^{2}, \quad g^{2}=\frac{\bar{g}^{2}}{Z_{\mathrm{F}}} .
$$

Actually, the effective action depends on both the background and the fluctuating gauge field, and the $n$-point functions can only be extracted from the functional depending on both fields [6]. Nevertheless, once all fluctuations are integrated out, the fluctuating field can be set to zero and the

\footnotetext{
${ }^{2}$ Already at this point, it is clear that $\lambda_{\phi, \Lambda}$ could also be chosen nonzero, which would only result in an unimportant change of the normalization of the functional integral.
} 
resulting effective action is gauge invariant. In general, the dependence of the effective action on both fields is needed for the RG flow. With the help of background-field identities, the dependences of the effective action on the fluctuating gauge field and the background field are related. A detailed record of the flow equations and results in the background field formalism, including the role of the gauge symmetry and Slavnov-Taylor identities, can be found in Refs. [6,7].

In the present work, we neglect possible differences between the RG flow for gauge couplings defined from the background-field effective action and from vertices of the fluctuating field $[8,9]$. This is perfectly justified in the limit of small gauge coupling which is of primary importance for this work. Here the lowest-order running is universal. By contrast, in the region of large coupling, our truncation of the gauge sector would anyway not be reliable if taken at face value, so that we abstain from resolving the gauge field running and $\eta_{\mathrm{F}}$ in this regime. In this region, we simply model the running of the gauge coupling in order to obtain a first glance at the $\chi \mathrm{SB}$ regime. Thereby, we assume that the influence of higher gluonic operators can be effectively accounted for by the increase of the gauge coupling. Although this certainly represents an oversimplification, let us stress that the details of the flow in the gauge sector are only of secondary importance for the issue addressed in this paper.

Inserting the truncation (4) into the exact RG flow equation for the effective average action, we find the following results. The scalar and fermion anomalous dimensions can be written as

$$
\begin{aligned}
\eta_{\phi}= & 4 v_{4} \kappa \lambda_{\phi}^{2} m_{2,2}^{4}\left(0,2 \kappa \lambda_{\phi} ; \eta_{\phi}\right) \\
& +4 N_{\mathrm{c}} v_{4} h^{2}\left[m_{4}^{(\mathrm{F}), 4}\left(\kappa h^{2} ; \eta_{\psi}\right)+\kappa h^{2} m_{2}^{(\mathrm{F}), 4}\left(\kappa h^{2} ; \eta_{\psi}\right)\right],
\end{aligned}
$$

$$
\begin{aligned}
\eta_{\psi}= & 2 C_{2}\left(N_{\mathrm{c}}\right) v_{4} g^{2}\left[(3-\xi) m_{1,2}^{(\mathrm{FB}), 4}\left(\kappa h^{2}, 0 ; \eta_{\psi}, \eta_{\mathrm{F}}\right)\right. \\
& \left.-3(1-\xi) \tilde{m}_{1,1}^{(\mathrm{FB}), 4}\left(\kappa h^{2}, 0 ; \eta_{\psi}, \eta_{\mathrm{F}}\right)\right] \\
& +v_{4} h^{2}\left[m_{1,2}^{(\mathrm{FB}), 4}\left(\kappa h^{2}, \epsilon+2 \kappa \lambda_{\phi} ; \eta_{\psi}, \eta_{\phi}\right)\right. \\
& \left.+m_{1,2}^{(\mathrm{FB}), 4}\left(\kappa h^{2}, \epsilon ; \eta_{\psi}, \eta_{\phi}\right)\right],
\end{aligned}
$$

where $v_{4}=1 /\left(32 \pi^{2}\right)$ and $C_{2}\left(N_{\mathrm{c}}\right)=\left(N_{\mathrm{c}}^{2}-1\right) /\left(2 N_{\mathrm{c}}\right)$. This representation is valid in the symmetric as well as in the $\chi \mathrm{SB}$ regime. In the former, $\kappa$ has to be set equal to zero, whereas $\epsilon=0$ has to be chosen in the latter. The various quantities denoted by $m$ are threshold functions which control the decoupling of massive modes for decreasing $k$; they also contain all dependencies on the precise choice of the cutoff function $R_{k}$. Their definitions and explicit representations can be found in Appendix A or in Ref. [4].

Equation (13) agrees with Refs. [4,11]. We also find agreement for the second line of Eq. (14), whereas the first line arises from the gauge-field sector (which has not been dealt with in Refs. $[4,11])$. As a further check, we note that in the perturbative small-coupling limit, where the threshold functions $m$ occurring above universally reduce to 1 , we obtain

$$
\left.\eta_{\phi}\right|_{\text {pert. }}=\frac{N_{\mathrm{c}}}{8 \pi^{2}} h^{2},\left.\quad \eta_{\psi}\right|_{\text {pert. }}=\xi \frac{C_{2}\left(N_{\mathrm{c}}\right)}{8 \pi^{2}} g^{2}+\frac{1}{16 \pi^{2}} h^{2},
$$

which agrees with the literature [12].

In the symmetric regime, the flow of the purely scalar sector can be summarized by

$\partial_{t} \epsilon=-\left(2-\eta_{\phi}\right) \epsilon-8 v_{4} \lambda_{\phi} l_{1}^{4}\left(\epsilon ; \eta_{\phi}\right)+8 N_{\mathrm{c}} v_{4} h^{2} l_{1}^{(\mathrm{F}), 4}\left(0 ; \eta_{\psi}\right)$,

$\partial_{t} \lambda_{\phi}=2 \eta_{\phi} \lambda_{\phi}+20 v_{4} \lambda_{\phi}^{2} l_{2}^{4}\left(\epsilon ; \eta_{\phi}\right)-8 N_{\mathrm{c}} v_{4} h^{4} l_{2}^{(\mathrm{F}), 4}\left(0 ; \eta_{\psi}\right)$,

whereas in the $\chi \mathrm{SB}$ regime, we find

$$
\begin{aligned}
\partial_{t} \kappa= & -\left(2+\eta_{\phi}\right) \kappa+2 v_{4} l_{1}^{4}\left(0 ; \eta_{\phi}\right)+6 v_{4} l_{1}^{4}\left(2 \kappa \lambda_{\phi} ; \eta_{\phi}\right) \\
& -8 N_{\mathrm{c}} v_{4} \frac{h^{2}}{\lambda_{\phi}} l_{1}^{(\mathrm{F}), 4}\left(\kappa h^{2} ; \eta_{\psi}\right), \\
\partial_{t} \lambda_{\phi}= & 2 \eta_{\phi} \lambda_{\phi}+2 v_{4} \lambda_{\phi}^{2} l_{2}^{4}\left(0 ; \eta_{\phi}\right)+18 v_{4} \lambda_{\phi}^{2} l_{2}^{4}\left(2 \kappa \lambda_{\phi} ; \eta_{\phi}\right) \\
& -8 N_{\mathrm{c}} v_{4} h^{4} l_{2}^{(\mathrm{F}), 4}\left(\kappa h^{2} ; \eta_{\psi}\right),
\end{aligned}
$$

in complete agreement with the results of Ref. [11]. Again, the quantities denoted by $l$ are threshold functions $[4,13]$. Now we turn to the flow of the Yukawa coupling, which is driven by all sectors of the system:

$$
\begin{aligned}
\partial_{t} h^{2}= & \left(2 \eta_{\psi}+\eta_{\phi}\right) h^{2}-4 v_{4} h^{4}\left[l_{1,1}^{(\mathrm{FB}), 4}\left(\kappa h^{2}, \epsilon ; \eta_{\psi}, \eta_{\phi}\right)\right. \\
& \left.-l_{1,1}^{(\mathrm{FB}), 4}\left(\kappa h^{2}, \epsilon+2 \kappa \lambda_{\phi} ; \eta_{\psi}, \eta_{\phi}\right)\right] \\
& -8(3+\xi) C_{2}\left(N_{\mathrm{c}}\right) v_{4} g^{2} h^{2} l_{1,1}^{(\mathrm{FB}), 4}\left(\kappa h^{2}, 0 ; \eta_{\psi}, \eta_{\mathrm{F}}\right),
\end{aligned}
$$

where we have to set $\kappa=0 \quad(\epsilon=0)$ in the symmetric $(\chi \mathrm{SB})$ regime. As a check, we take a look at the perturbative limit,

$$
\left.\partial_{t} h^{2}\right|_{\text {pert. }}=\frac{N_{\mathrm{c}}+1}{8 \pi^{2}} h^{4}-\frac{3 C_{2}\left(N_{\mathrm{c}}\right)}{4 \pi^{2}} g^{2} h^{2},
$$

where we rediscover known results and also observe that the gauge-parameter $\xi$ dependence has dropped out as it should.

A crucial ingredient is the flow of the fermion selfinteraction, which - in a dimensionful representation-can be written as

$$
\begin{aligned}
& \partial_{t} \bar{\lambda}_{\sigma}=\frac{Z_{\psi}^{2}}{k^{2}}\left[\beta_{\lambda_{\sigma}}^{g^{4}} g^{4}+\beta_{\lambda_{\sigma}}^{h^{4}} h^{4}\right], \\
& \beta_{\bar{\lambda}_{\sigma}}^{g^{4}}:=-6 \frac{\left(N_{\mathrm{c}}+2\right)\left(N_{\mathrm{c}}-1\right)}{N_{\mathrm{c}}^{2}} C_{2}\left(N_{\mathrm{c}}\right) v_{4} \widetilde{l}_{1,2}^{(\mathrm{FB}), 4}\left(\kappa h^{2}, 0 ; \eta_{\psi}, \eta_{\mathrm{F}}\right), \\
& \beta_{\bar{\lambda}_{\sigma}}^{h^{4}}:=\left(\frac{2}{N_{\mathrm{c}}}+1\right) v_{4} \widetilde{l}_{1,1,1}^{(\mathrm{FBB}), 4}\left(\kappa h^{2}, \epsilon, \epsilon+2 \kappa \lambda_{\phi} ; \eta_{\psi}, \eta_{\phi}\right) .
\end{aligned}
$$

Here we neglected terms $\sim \kappa$ which arise only in the broken regime but are suppressed therein owing to simultaneously 
occurring threshold functions (these terms are similar to the last term in square brackets in Eq. (13), which has hardly any effect on the results either). In Eq. (22) as well as in all equations above, we neglected terms of order $\bar{\lambda}_{\sigma}$ on the right-hand side, because $\bar{\lambda}_{\sigma}=0$ will finally be guaranteed on all scales as discussed below. Furthermore, we have chosen the same Fierz transformations in the Dirac algebra as in Ref. [2] and decomposed the possible color structures of the fourfermion interaction into a color singlet $(\mathrm{S}-\mathrm{P})_{\mathrm{S}}$ and color $N_{\mathrm{c}}^{2}-1$-plets $(\mathrm{S}-\mathrm{P})_{N_{\mathrm{c}}^{2}-1},(\mathrm{~V})_{N_{\mathrm{c}}^{2}-1}$. In the present work, we focus on the $(\mathrm{S}-\mathrm{P})_{\mathrm{S}}$ term; in principle, the $(\mathrm{V})_{N_{\mathrm{c}}^{2}-1}$ term could be absorbed into a $k$-dependent transformation of the non-Abelian gauge field in the same way as suggested in Ref. [2] for the Abelian case. ${ }^{3}$

As mentioned above, there is a certain redundancy in the parametrization of the effective action $\Gamma_{k}$ owing to possible different choices of partial bosonization of the four-fermion interaction. From a different viewpoint, this redundancy corresponds to the possible mixing of fields or composite operators with identical quantum numbers. We remove this redundancy in the present truncation with the aid of the following $k$-dependent transformation of the scalar field ("fermionboson translation"):

$$
\begin{gathered}
\partial_{t} \phi_{k}(q)=-\left(\bar{\psi}_{\mathrm{L}} \psi_{\mathrm{R}}\right)(q) \partial_{t} \alpha_{k}(q)+\phi_{k}(q) \partial_{t} \beta_{k}(q), \\
\partial_{t} \phi_{k}^{*}(q)=\left(\bar{\psi}_{\mathrm{R}} \psi_{\mathrm{L}}\right)(-q) \partial_{t} \alpha_{k}(q)+\phi_{k}^{*}(q) \partial_{t} \beta_{k}(q),
\end{gathered}
$$

with a priori arbitrary functions $\alpha_{k}(q)$ and $\beta_{k}(q)$. Upon this transformation, the flow equations given above receive additional contributions $\sim \alpha_{k}(q), \beta_{k}(q)$ according to

$$
\partial_{t} \Gamma_{k}=\partial_{t} \Gamma_{k \mid \phi_{k}, \phi_{k}^{*}}+\int \frac{\delta \Gamma_{k}}{\delta \phi_{k}} \partial_{t} \phi_{k}+\int \frac{\delta \Gamma_{k}}{\delta \phi_{k}^{*}} \partial_{t} \phi_{k}^{*} .
$$

As described in more detail in Ref. [2], these functions can be uniquely determined by demanding for (i) $\partial_{t} \bar{\lambda}_{\sigma}\left(q^{2}\right)$ to vanish for all $k$ and $q^{2}$, where the momentum dependence of $\bar{\lambda}_{\sigma}$ has been studied in the $s$ channel for simplicity, $\bar{\lambda}_{\sigma}\left(q^{2}\right)$ $\equiv \bar{\lambda}_{\sigma}\left(s=q^{2}\right)$, (ii) the Yukawa coupling $\bar{h}$ to be momentum independent, and (iii) $\partial_{t} Z_{\phi}\left(q^{2}=k^{2}\right)=-\eta_{\phi} Z_{\phi}$ in order to render the approximation of a momentum-independent $Z_{\phi}$ self-consistent. Condition (i) together with the initial condition (7) guarantees that no four-fermion interaction of this type is generated under the flow; this interaction is bosonized into the scalar sector at all scales $k$. Condition (ii) guarantees

\footnotetext{
${ }^{3}$ By neglecting some of the four-fermion interactions, our quantitative result will depend slightly on the choice of the Fierz decomposition. Using a "fermion-boson translation" to be described in the following, this dependence can be removed in a larger truncation, as was recently shown in Ref. [10]. However, we checked explicitly that quantitative results in another natural Fierz decomposition involving $(\mathrm{S}-\mathrm{P})_{\mathrm{S}},(\mathrm{V})_{\mathrm{S}}$ and $(\mathrm{V})_{N_{\mathrm{c}}{ }^{2}-1}$ differ from the present ones only on the $1 \%$ level.
}

the fermion mass generated by $\chi \mathrm{SB}$ is also momentum independent, so that the couplings in the $\chi \mathrm{SB}$ regime have a direct physical interpretation.

The field transformation (23) affects also the scalar couplings, and we obtain, in the symmetric regime,

$$
\begin{gathered}
\partial_{t} \epsilon=\left.\partial_{t} \epsilon\right|_{\phi_{k}}+2 \frac{\epsilon(1+\epsilon)}{h^{2}}\left(1+(1+\epsilon) Q_{\sigma}\right)\left(\beta_{\bar{\lambda}_{\sigma}}^{g^{4}} g^{4}+\beta_{\bar{\lambda}_{\sigma}}^{h^{4}} h^{4}\right), \\
\partial_{t} h^{2}=\left.\partial_{t} h^{2}\right|_{\phi_{k}}+2\left(1+2 \epsilon+Q_{\sigma}(1+\epsilon)^{2}\right)\left(\beta_{\bar{\lambda}_{\sigma}}^{g^{4}} g^{4}+\beta_{\bar{\lambda}_{\sigma}}^{h^{4}} h^{4}\right),
\end{gathered}
$$

where the corresponding first terms on the right-hand sides denote the flow equations for fixed fields as given above in Eqs. (16) and (20). In the $\chi \mathrm{SB}$ regime, we find similarly

$$
\begin{aligned}
\partial_{t} \kappa= & \left.\partial_{t} \kappa\right|_{\phi_{k}}+2 \frac{\kappa\left(1-\kappa \lambda_{\phi}\right)}{h^{2}}\left(1+\left(1-\kappa \lambda_{\phi}\right) Q_{\sigma}\right) \\
& \times\left(\beta_{\lambda_{\sigma}}^{g^{4}} g^{4}+\beta_{\bar{\lambda}_{\sigma}}^{h^{4}} h^{4}\right), \\
\partial_{t} h^{2}= & \left.\partial_{t} h^{2}\right|_{\phi_{k}}+2\left(1-2 \kappa \lambda_{\phi}+Q_{\sigma}\left(1-\kappa \lambda_{\phi}\right)^{2}\right) \\
& \times\left(\beta_{\bar{\lambda}_{\sigma}^{g}}^{g^{4}} g^{4}+\beta_{\bar{\lambda}_{\sigma}}^{h^{4}} h^{4}\right) .
\end{aligned}
$$

Defining $\quad \Delta \bar{\lambda}_{\sigma}:=\bar{\lambda}_{\sigma}\left(k^{2}\right)-\bar{\lambda}_{\sigma}(0)$, the quantity $Q_{\sigma}$ $\equiv \partial_{t} \Delta \bar{\lambda}_{\sigma} / \partial_{t} \bar{\lambda}_{\sigma}(0)$ measures the suppression of $\bar{\lambda}_{\sigma}(s)$ for large external momenta. Without an explicit computation, we may conclude that this suppression implies $Q_{\sigma}<0$, in agreement with unitarity; furthermore, if the flow is in the $\chi \mathrm{SB}$ regime, the fermions become massive, and nonpointlike four-fermion interactions in the $s$ channel will be suppressed by the inverse fermion mass squared. ${ }^{4}$ Therefore, we model $Q_{\sigma}$ by the ansatz

$$
Q_{\sigma}=Q_{\sigma}^{0} m_{1,2}^{(\mathrm{FB}), 4}\left(\kappa h^{2}, 0, \eta_{\psi}, \eta_{F}\right), \quad Q_{\sigma}^{0}=\mathrm{const}<0,
$$

where we have introduced a threshold function with the appropriate decoupling properties for massive fermions. The qualitative results are independent of the precise choice of $Q_{\sigma}$, and it is reassuring to observe a quantitative independence of the IR observables on the precise value for $Q_{\sigma}^{0}$ (e.g., $\left.Q_{\sigma}^{0} \simeq-0.1\right)$.

The field transformations (23) also modify the equation for $\lambda_{\phi}$ via the terms $\sim \partial_{t} \beta_{k}$. In the pointlike limit $\left(q^{2}\right.$ $=0$ ), the modified running is given by

\footnotetext{
${ }^{4}$ This can be inferred from the heavy-fermion limit of the twogluon/scalar-exchange box diagram where the internal fermion propagators become pointlike, $\sim 1 / m_{\mathrm{f}}$.
} 


$$
\begin{aligned}
\partial_{t} \lambda_{\phi}= & \left.\partial_{t} \lambda_{\phi}\right|_{\phi_{k}} \\
& +4 \frac{\lambda_{\phi}}{h^{2}}(1+\epsilon)\left[1+(1+\epsilon) Q_{\sigma}\right]\left(\beta_{\lambda_{\sigma}}^{\frac{g^{4}}{}} g^{4}+\beta_{\lambda_{\sigma}}^{h^{4}} h^{4}\right) .
\end{aligned}
$$

It will turn out that the modification of the flow of $\lambda_{\phi}$ is also quantitatively irrelevant, whereas the modifications displayed in Eqs. (25) and (26) are of crucial importance.

\section{BOUND-STATE FIXED POINT}

The universal features of spontaneous $\chi \mathrm{SB}$ in the $\mathrm{QCD}$ domain that will be quantitatively analyzed in the next section can be traced back to the occurrence of a fixed point for the scalar couplings. This fixed point is infrared attractive as long as the gauge coupling remains weak and can be associated with a bound state [2].

The fixed-point structure can conveniently be analyzed with the help of the coupling

$$
\tilde{\epsilon}=\frac{\epsilon}{h^{2}}=\frac{Z_{\psi}^{2} \bar{m}^{2}}{k^{2} \bar{h}^{2}} .
$$

Since we are interested in the domain of weak gauge coupling, for simplicity we can neglect the anomalous dimensions in the following. In this approximation and choosing the gauge parameter $\xi=0$ (background Landau gauge), the flow of $\tilde{\epsilon}$ yields

$$
\begin{aligned}
\partial_{t} \tilde{\epsilon}= & 8 N_{\mathrm{c}} v_{4} l_{1}^{(\mathrm{F}), 4}-8 v_{4} l_{1}^{4}(\epsilon) \frac{\lambda_{\phi}}{h^{2}} \\
& -\left(2-24 C_{2}\left(N_{\mathrm{c}}\right) v_{4} l_{1,1}^{(\mathrm{FB}), 4} g^{2}\right) \tilde{\epsilon}-2\left(\beta_{\lambda_{\sigma}}^{g^{4}} g^{4}+\beta_{\bar{\lambda}_{\sigma}}^{h^{4}} h^{4}\right) \tilde{\epsilon}^{2} .
\end{aligned}
$$

(Here, all arguments of the threshold functions which are not displayed are assumed to be equal to zero; therefore, threshold functions without any argument are simply numbers which depend on the details of the regularization.) If the scalar field is auxiliary at the UV scale as in the QCD context, its wave function renormalization is very small initially, $Z_{\phi} \ll 1$, so that the dimensionless renormalized mass is very large, $\epsilon \gg 1$. In this case, scalar fluctuations are suppressed and the threshold functions depending on $\epsilon$ vanish; the righthand side of Eq. (30) describes a parabola in the variable $\tilde{\epsilon}$, and we find two positive fixed points, $0<\tilde{\epsilon}_{1}^{*}<\tilde{\epsilon}_{2}^{*}$, where $\tilde{\epsilon}_{1}^{*}$ is UV attractive but IR unstable, and $\tilde{\epsilon}_{2}^{*}$ is an IR stable fixed point (see Fig. 2, solid line). It can be shown that $\tilde{\epsilon}_{1}^{*}$ corresponds to the inverse of the critical coupling of the NJL model, so that our flow describes a model with strong fourfermion interaction if we choose UV initial conditions with $\tilde{\epsilon}_{\Lambda}<\tilde{\epsilon}_{1}^{*}$ to the left of the first fixed point (see, e.g., Ref. [14] for a detailed analysis of the phase structure in the Abelian case). For this choice, the system is not in the QCD domain

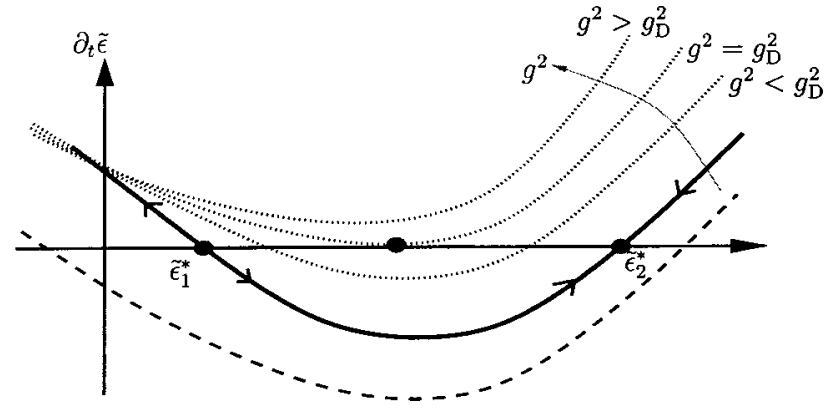

FIG. 2. Flow of $\tilde{\epsilon}$ according to Eq. (30) (schematic plot): the solid line corresponds to a QCD scenario at weak gauge coupling; the arrows indicate the direction of the flow towards the infrared. The dashed line corresponds to a system with fundamental scalar, $\left.Z_{\phi}\right|_{k=\Lambda}=1, \epsilon \lesssim 1$, and strong scalar self-interaction. The dotted lines exhibit the destabilization of the bound-state fixed point by the increasing gauge coupling.

but approaches chiral symmetry breaking $(\tilde{\epsilon}<0)$ in a perturbatively accessible way $(\mathrm{P} \chi \mathrm{SB})$.

In this section, we concentrate on those initial values which release the system to the right of the first fixed point, $\tilde{\epsilon}_{\Lambda}>\tilde{\epsilon}_{1}^{*}$, i.e., which are weakly coupled in the NJL language. This will be the range of the QCD universality class. As the system evolves, it flows towards the second fixed point $\widetilde{\epsilon}_{2}^{*}$, which then governs the evolution over many scales. Here, the system "loses its memory" of the initial conditions; in particular, it is of no relevance whether we start with $\tilde{\epsilon}_{1}^{*}$ $<\tilde{\epsilon}_{\Lambda}<\tilde{\epsilon}_{2}^{*}$ or $\tilde{\epsilon}_{\Lambda}>\tilde{\epsilon}_{2}^{*}$. The evolution towards and in the IR is universally governed by this fixed point $\tilde{\epsilon}_{2}^{*}$, which can be shown to be associated with a fermion-antifermion bound state; e.g., in QED, the properties of the scalar field at this fixed point correspond to those of positronium [2].

Before we elucidate the fixed-point properties further, let us briefly mention that its existence can be generalized to the case of a scalar field describing a fundamental particle in the UV (a Yukawa model with gauged fermions rather than QCD). In this case, we have $Z_{\phi}=1$ and $\epsilon \simeq \mathcal{O}(1)$ at the UV scale. Now the second term in Eq. (30) can become important, in particular for a large $\phi^{4}$ coupling $\lambda_{\phi}$ and/or small $h^{2}$. When discussing the right-hand side of Eq. (30) for fixed $g$, $h, \lambda_{\phi}$, one should keep in mind that these couplings may change with $k$. For large $\lambda_{\phi} / h^{2}$, the $\tilde{\epsilon}$ parabola is lowered and the first fixed point can move to negative values, $\tilde{\epsilon}_{1}^{*}$ $<0$ (see Fig. 2, dashed line). In this case, we can release the system even in the broken regime at the UV scale, $\tilde{\epsilon}, \epsilon<0$, but it still evolves towards the bound-state fixed point $\tilde{\epsilon}_{2}^{*}$. In comparison with Fig. 1, this corresponds to initial values $\bar{m}_{\mathrm{c}}^{2}<\bar{m}_{\Lambda}^{2}<0$. Physically, such a scenario describes a system involving fundamental scalars, fermions and gauge fields, where the scalar sector is initially weakly coupled to the fermions. If we start in the broken regime, scalar fluctuations will drive the system towards the symmetric regime before the fermion-gauge-field interactions induce sizable boundstate effects which can exert an influence on the scalar sector. In this scenario, the first fixed point $\tilde{\epsilon}_{1}^{*}<0$ is a measure of 
the strength of the initial effective coupling between scalars and fermions. For strong effective coupling, $\tilde{\epsilon}_{\Lambda}<\tilde{\epsilon}_{1}^{*}$, an initial negative scalar mass of the order of the cutoff, $\left.\bar{m}^{2}\right|_{k=\Lambda}$ $\simeq-\mathcal{O}\left(\Lambda^{2}\right)$ will induce a vacuum expectation value and a fermion mass of the same order, in agreement with naive expectations. But at weak effective coupling, e.g., $h^{2}$ $\sim \mathcal{O}(1), \lambda_{\phi} \simeq 100$ and $\tilde{\epsilon}_{1}^{*}<\tilde{\epsilon}_{\Lambda}<0$, the system can still start with an initial negative scalar mass $\left.\bar{m}^{2}\right|_{k=\Lambda} \simeq-\mathcal{O}\left(\Lambda^{2}\right)$, but finally run into the bound-state fixed point. As an important result, the vacuum expectation value and the fermion mass after symmetry breaking can easily be orders of magnitude smaller than the UV scale, as exhibited in Fig. 1 in Sec. I. We conclude that all systems with $\tilde{\epsilon}_{\Lambda}>\tilde{\epsilon}_{1}^{*}$ belong to the QCD universality class.

Let us now turn to the properties of the system at the bound-state fixed point. The crucial observation is that not only $\tilde{\epsilon}$ but also all dimensionless scalar couplings approach fixed points. In the general case, the fixed-point values depend in a complicated form on all parameters of the system. However, in the limit $\epsilon \gg 1$ (QCD-like), we can find analytic expressions that satisfy the fixed-point conditions $\partial_{t}\left(\epsilon, h^{2}, \lambda_{\phi}\right)=0$ to leading order:

$$
\begin{aligned}
\epsilon^{*} & \simeq \frac{2}{\left|Q_{\sigma}\right|}, \\
\left(h^{*}\right)^{2} & \simeq \frac{2\left|\beta_{\lambda_{\sigma}}^{g^{4}}\right| g^{4}}{\left|Q_{\sigma}\right|} \\
& =\frac{12}{\left|Q_{\sigma}\right|} \frac{C_{2}\left(N_{\mathrm{c}}\right)\left(N_{\mathrm{c}}+2\right)\left(N_{\mathrm{c}}-1\right)}{N_{\mathrm{c}}^{2}} v_{4} l_{1,2}^{(\mathrm{FB}), 4} g^{4}, \\
\lambda_{\phi}^{*} & \simeq \frac{N_{\mathrm{c}}\left(h^{*}\right)^{4}}{6 C_{2}\left(N_{\mathrm{c}}\right) g^{2}} .
\end{aligned}
$$

From the first equation, we read off that the approximation $\epsilon \gg 1$ is equivalent to assuming $\left|Q_{\sigma}\right| \ll 1$, which is roughly fulfilled in our numerical study with our choice of $Q_{\sigma}^{0}$ $=-0.1$.

The remarkable properties of the IR fixed point become apparent when considering the renormalized scalar mass, $m^{2}=\epsilon k^{2}$. Since $\epsilon \rightarrow \epsilon^{*}$, the scalar mass simply decreases with the scale $k$, so that it is only natural to obtain small masses $m^{2} \ll \bar{m}_{\Lambda}^{2}$ for small scale ratios $k \ll \Lambda$. In other words, even if we start with a scalar mass of the order of the cutoff, $\left.\bar{m}^{2}\right|_{k=\Lambda} \sim \Lambda^{2}$, no fine tuning will be necessary to obtain small mass values at low-energy scales, as long as the running is controlled by the bound-state fixed point.

In order to approach the $\chi \mathrm{SB}$ regime, the bound-state fixed point has to be destabilized; otherwise, the system will remain in the symmetric regime as is the case in QED. In QCD, this destabilization arises from the increase of the gauge coupling towards the infrared [15]. From the third and last term of Eq. (30), it is obvious that an increasing gauge coupling lifts the $\partial_{t} \tilde{\epsilon}$ parabola (see Fig. 2, dotted lines). For some value $g_{\mathrm{D}}^{2}$ of the gauge coupling, the two fixed-points in $\tilde{\epsilon}$ will be degenerate, so that there is no fixed point at all for all $g^{2}>g_{\mathrm{D}}^{2}$. The beta function $\partial_{t} \tilde{\epsilon}$ is then strictly positive, which drives the system towards the $\chi \mathrm{SB}$ regime.

In the limit $\epsilon \gg 1$, the critical gauge coupling of fixedpoint degeneracy $g_{\mathrm{D}}^{2}$ can be computed analytically, and we find

$$
g_{\mathrm{D}}^{2} \simeq \frac{16}{3} \pi^{2} \frac{N_{\mathrm{c}}}{N_{\mathrm{c}}-1}\left(\sqrt{1+\frac{1}{N_{\mathrm{c}}+1}}-1\right) \simeq \frac{4}{3} \pi^{2} \frac{1}{C_{2}\left(N_{\mathrm{c}}\right)},
$$

where we have used linear cutoff functions [16] for which $l_{1,2}^{(\mathrm{FB}), 4}=3 / 2$. For instance, for $\mathrm{SU}(3)$ we get $\alpha_{\mathrm{D}}=g_{\mathrm{D}}^{2} / 4 \pi$ $\simeq \pi / 4$, which is in the nonperturbative domain, as expected. ${ }^{5}$ As soon as $g^{2}$ exceeds $g_{\mathrm{D}}^{2}$, the running of the scalar couplings is no longer protected by the bound-state fixed point. Here all couplings are expected to run fast, being strongly influenced by the details of the increase of the gauge coupling. Of course, owing to strong coupling, many higherorder operators can acquire large anomalous dimensions and contribute to the dynamics of the symmetry-breaking transition. Our truncation should be understood as the minimal lowest-order approximation in this regime, but gives already a remarkably consistent (but not necessarily complete) picture. Once chiral symmetry is broken, the fermions decouple and the fermionic and (most of the) scalar flow essentially stops.

The scenario discussed here finally explains why the IR values of the scalar and fermionic couplings inherit their order of magnitude from the QCD scale $\Lambda_{\mathrm{QCD}}$ as they should, whereas particularly the details of the scalar sector at the UV scale are of no relevance, owing to the fixed-point structure inducing QCD universality.

\section{NUMERICAL RESULTS}

In the following, we concentrate on the set of theories that belong to the QCD universality class. In order to illustrate how universality arises from the presence of the bound-state fixed point, we initiate our flows at a GUT-like scale of $\Lambda$ $=10^{15} \mathrm{GeV}$, where the gauge coupling is weak and increases only logarithmically towards the infrared. Therefore, the bound-state fixed point exists over a wide range of scales. As discussed before, hardly any dependence on the specific initial values for the scalar potential and the Yukawa coupling remains because of the fixed point, as we will demonstrate quantitatively in the following.

For illustrative purposes, we concentrate here on QCDlike scenarios where the scalar is auxiliary at the UV scale, and explore this parameter space using the natural choice

\footnotetext{
${ }^{5}$ Strictly speaking, the value of $g_{\mathrm{D}}^{2}$ is not a physical quantity and depends on the choice of the cutoff function, i.e., the regularization scheme. This is only natural, since the running of the coupling itself also depends on the regularization. The scheme dependence, however, cancels out in physical quantities.
} 

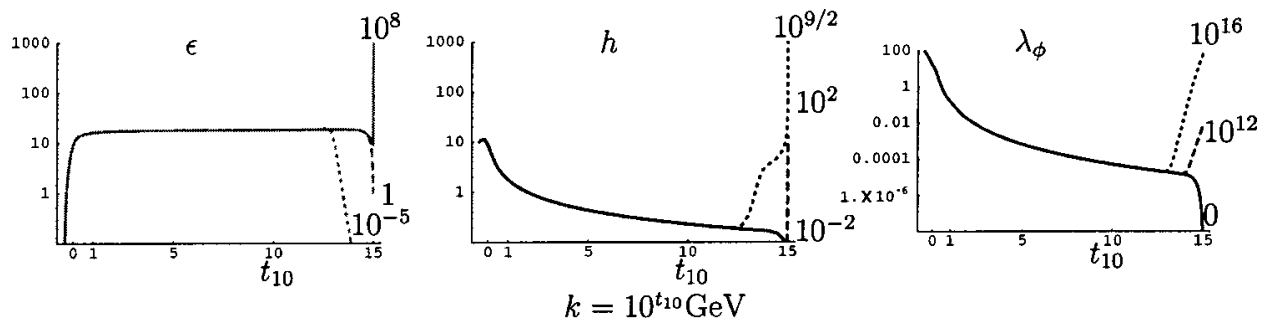

FIG. 3. Flow of $\epsilon, h$ and $\lambda_{\phi}$ in the symmetric regime according to Eqs. (16), (17), (20), and (25). The solid lines correspond to the reference set (33), whereas the dotted and dashed lines represent the flows for strongly differing initial values as indicated. The insensitivity with respect to the choice of initial conditions is clearly visible. On the horizontal axis, the exponent $t_{10}$ is used for the scale $k$ $=10^{t} 10 \mathrm{GeV}$.

given by Eq. (8) as a reference; to be precise, we use the reference set

$$
\begin{gathered}
\left.\bar{m}^{2}\right|_{k=\Lambda}=\Lambda^{2},\left.\quad \bar{\lambda}_{\phi}\right|_{k=\Lambda}=0,\left.\quad Z_{\phi}\right|_{k=\Lambda}=10^{-8}, \\
\left.\bar{h}^{2}\right|_{k=\Lambda}=10^{-12},\left.\quad \Leftrightarrow \epsilon\right|_{\Lambda}=10^{8},\left.\quad \lambda_{\phi}\right|_{\Lambda}=0,\left.\quad h\right|_{\Lambda}=10^{-2},
\end{gathered}
$$

in our numerical studies. In all computations, we use linear cutoff functions proposed in Ref. [16] for which the threshold functions can be determined analytically (see Appendix A). We plot the flows of the renormalized dimensionless couplings $\epsilon, h$ and $\lambda_{\phi}$ in Fig. 3 for the symmetric regime. The reference set (33) is depicted as solid lines, whereas the dashed and dotted lines correspond to initial values which deviate from the reference set (33) by many orders of magnitude for the corresponding couplings.

As long as we start in the range of attraction of the boundstate fixed point, we can obviously vary the initial values for the scalar couplings over many orders of magnitude without any appreciable effect. The system quickly approaches the bound-state fixed point, where the initial values of the couplings become unimportant. In particular, the scalar mass, which is allowed to be of the order of the cutoff or even much larger at $k=\Lambda$, runs to small values $\sim k$ while the system is governed by the bound-state fixed point. No fine tuning is necessary for this. ${ }^{6}$ Let us stress once more that these features of universality are not restricted to the reference set (33) and the variations thereof. They can also be found in Yukawa models with a fundamental scalar $\left(\left.Z_{\phi}\right|_{k=\Lambda}=1\right)$ and even if we start in the broken regime at the UV scale (see Fig. 1).

At the bound-state fixed point, the couplings are modulated only by the logarithmically slow increase of the gauge coupling. Incidentally, the modulation of $\tilde{\epsilon}=\epsilon / h^{2}$ is completely carried by $h$, whereas $\epsilon$ stays fixed. This agrees with our analytical fixed-point values found in Eq. (31). A rapid

\footnotetext{
${ }^{6}$ As a fairly weak condition, we only have to ensure that the initial scalar couplings are such that no strong four-fermion interaction is implicitly induced by the initial values; in this case, the system starts to the left of the first fixed point $\tilde{\epsilon}_{1}^{*}$ and rather resembles a non-Abelian gauged NJL model with $\mathrm{P} \chi \mathrm{SB}$.
}

change for the couplings in Fig. 3 is visible after $g^{2}$ exceeds $g_{\mathrm{D}}^{2}$ and the bound-state fixed point has disappeared $\left(t_{10}\right.$ $\lesssim 1)$.

The behavior of the system changes rapidly after the gauge coupling has grown large. For $g^{2}>g_{\mathrm{D}}^{2}$, the boundstate fixed point vanishes and all couplings start to run fast. The system necessarily runs into the $\chi \mathrm{SB}$ regime where the scalars develop a vacuum expectation value and the fermions acquire a mass

$$
m_{\mathrm{f}}^{2}=\lim _{k \rightarrow 0} k^{2} \kappa h^{2} \equiv\left(h \sigma_{\mathrm{R}}\right)^{2},
$$

where $\sigma_{\mathrm{R}}=\lim _{k \rightarrow 0} \sqrt{Z_{\phi} \rho_{0}}$ denotes the renormalized expectation value of the scalar field.

This leads to a decoupling of the fermions, and, consequently, fermion-boson translation is "switched off." Also the flow of the Yukawa coupling stops, the scalar and fermion anomalous dimensions approach zero, and $\kappa$ runs according to its trivial mass scaling, $\kappa \sim 1 / k^{2}$, so that $m_{\mathrm{f}}$ approaches a constant value.

Whereas the qualitative picture is rather independent of the details of the running gauge coupling, quantitative results are highly sensitive to the flow of the gauge sector. This is because a finite amount of "RG time" passes from the disappearance of the bound-state fixed point to the transition into the $\chi \mathrm{SB}$ regime. In between, the running of the gauge coupling exerts a strong influence on all other couplings which are no longer protected by any fixed point. A purely perturbative running of the gauge coupling turns out to be insufficient for the present purpose, since the (unphysical) Landau pole destabilizes the system in the infrared.

For definiteness, let us consider a running coupling governed by the beta function

$$
\begin{aligned}
\partial_{t} g^{2}= & \beta_{g^{2}}=\eta_{\mathrm{F}} g^{2}=-2\left(b_{0} \frac{g^{4}}{16 \pi^{2}}+b_{1} \frac{g^{6}}{\left(16 \pi^{2}\right)^{2}}\right) \\
& \times\left[1-\exp \left(\frac{1}{\alpha_{*}}-\frac{1}{\frac{g^{2}}{4 \pi}}\right)\right]^{s}, \\
b_{0}= & \frac{11}{3} N_{\mathrm{c}}-\frac{2}{3} N_{\mathrm{f}}, \quad b_{1}=\frac{34}{3} N_{\mathrm{c}}^{2}-\frac{10}{3} N_{\mathrm{c}} N_{\mathrm{f}}-2 C_{2}\left(N_{\mathrm{c}}\right) N_{\mathrm{f}}
\end{aligned}
$$



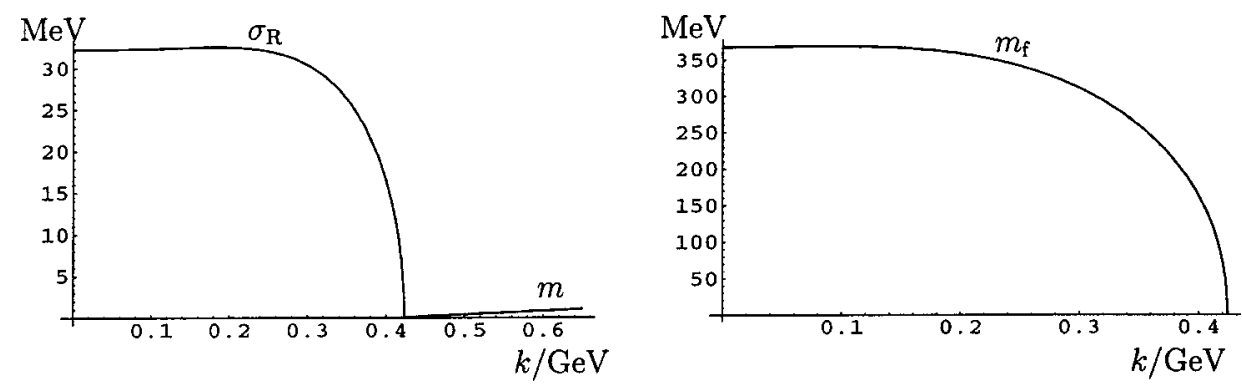

FIG. 4. Flow of the scalar mass $m$, the scalar VEV $\sigma_{\mathrm{R}}$, and the constituent quark mass $m_{\mathrm{f}}$ close to and in the $\chi \mathrm{SB}$ regime, using the reference set (33). For the particular choice for the running of the gauge coupling according to Eq. (35) with $\alpha_{*}=2.5$, the transition occurs at $k_{\chi \mathrm{SB}} \simeq 423 \mathrm{MeV}$.

for our numerical studies. In the UV, this beta function exhibits an accurate two-loop perturbative behavior, whereas the coupling runs to a fixed point $\alpha_{\mathrm{s}} \equiv g^{2} /(4 \pi) \rightarrow \alpha_{*}$ in the IR for $k \rightarrow 0$. In the first place, the infrared fixed point is convenient for numerical purposes, since it does not lead to artificial IR instabilities. Moreover, an infrared fixed point for a mass-scale-dependent running coupling is compatible with the expected mass gap in Yang-Mills theory. Below this mass gap, all gauge field fluctuations decouple from the flow and can no longer drive the flow of the coupling. Different beta functions with and without infrared fixed points are studied in Appendix B. It turns out that, though the infrared properties such as the constituent quark mass depend quantitatively on the choice of the beta function as expected, the universal features discussed in the following remain untouched. This underlines our observation that the detailed understanding of the flow for the region of strong gauge coupling is not essential for the overall picture.

In combination with Eq. (35), the system of flow equations is now closed and provides us with an answer for the (truncated) quantum effective action, once we specify all parameters and initial values. We have investigated $\mathrm{SU}\left(N_{\mathrm{c}}\right.$ $=3$ ) gauge theory with initial value $g(\Lambda)$ chosen such that $\alpha_{\text {s }}$ acquires its physical value at the Z-boson mass, $\alpha_{\mathrm{s}}\left(M_{Z}\right)$ $\simeq 0.117$. We work in the background Landau gauge, $\xi=0$, which is known to be a fixed point of the renormalization flow in the gauge sector $[17,18]$. If we had an exact flow equation at our disposal this choice would fix the system completely.

In our truncation, however, we have the parameter $Q_{\sigma}^{0}$, in addition to the Yang-Mills beta function, which characterizes our ignorance of the exact flow. The quantity $Q_{\sigma}^{0}$ measuring the momentum suppression of the four-fermion interaction will be set to $Q_{\sigma}^{0}=-0.1$, in agreement with our considerations given above. It turns out that the infrared properties of the system are only weakly dependent on this parameter and on $\xi$ (see below), which substantiates our truncation. Furthermore, we choose $\alpha_{*}$ to be of order 1, but not too close to $g_{\mathrm{D}}^{2} /(4 \pi)$ in order to avoid pathologies: $\alpha_{*}=2.5$.

For this concrete scenario, the transition to the $\chi \mathrm{SB}$ regime occurs at $k_{\chi \mathrm{SB}} \simeq 423 \mathrm{MeV}$. The renormalized scalar mass slightly above $k_{\chi \mathrm{SB}}$ and the VEV of the scalar field below $k_{\chi \text { SB }}$ are depicted in Fig. 4 (left panel). According to Eq. (34), we find a constituent quark mass of $m_{\mathrm{f}}$ $\simeq 371 \mathrm{MeV}$ as shown in Fig. 4 (right panel). Of course, these numbers depend strongly on the details of the Yang-Mills beta function for strong coupling $\alpha_{\mathrm{s}} \sim 1$; various other examples are discussed in Appendix B. Finally, the running of $\lambda_{\phi}, h^{2}$ and the scalar and fermionic wave function renormalizations is collected in Fig. 5.

Focusing on low-energy QCD-like aspects of our truncated system, it is also remarkable that (apart form the scalar couplings) the choice of $Q_{\sigma}^{0}$ has little effect on infrared prop-
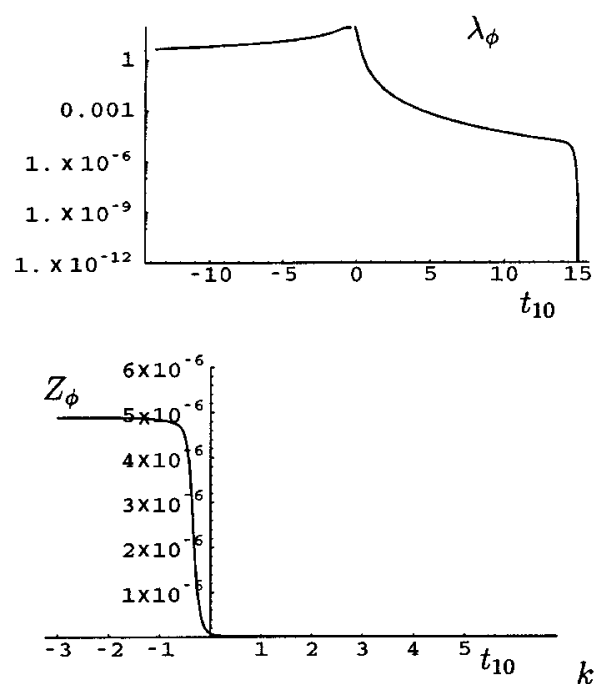
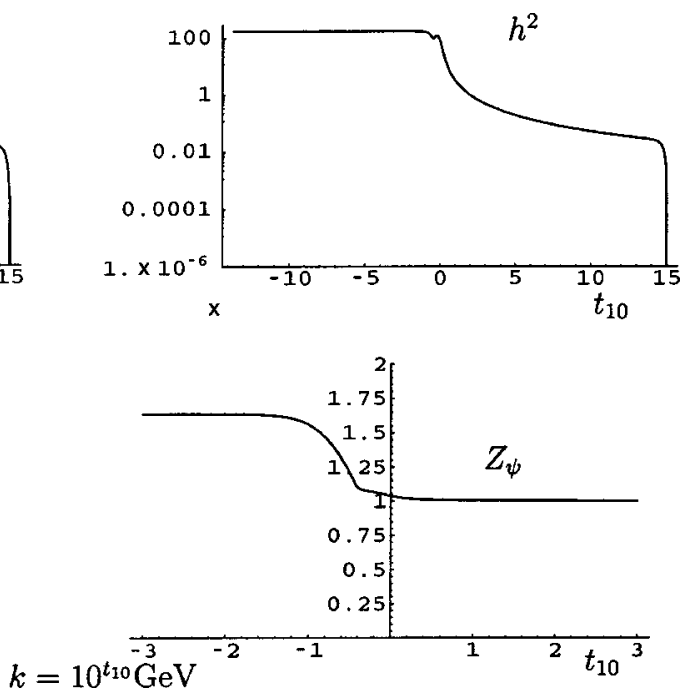

FIG. 5. Flow of $\lambda_{\phi}, h^{2}$, and the wave function renormalizations $Z_{\phi}$ and $Z_{\psi}$ over the complete range of scales for the reference set (33). The rapid change of all couplings near $t_{10}=\log _{10} k_{\chi \mathrm{SB}} / \Lambda$ $\simeq-0.5$ is visible. Whereas $h^{2}$, $Z_{\phi}$, and $Z_{\psi}$ approach fixed points in the deep infrared owing to decoupling, $\lambda_{\phi}$ decreases logarithmically owing to a massless "eta" in absence of the axial anomaly. 
erties of the system: varying $Q_{\sigma}^{0}$ between $-0.5 \ldots 0.001$ changes $k_{\chi \mathrm{SB}}$ or $m_{\mathrm{f}}$ only at the level of less than $10 \%$. This is reassuring and in contrast to the strong $Q_{\sigma}^{0}$ dependence of the bound-state fixed-point values of $\epsilon_{*}$ and $h_{*}$. The variations of the infrared properties are similarly small for changes in the gauge parameter in the interval $\xi=0 \ldots 2$.

To summarize, a large class of QCD-like theories including a scalar degree of freedom belong to the QCD universality class owing to an attractive infrared fixed point present for weak gauge coupling. Even before the gauge coupling becomes strong, all theories in this universality class are indistinguishable at low energies. They exhibit an identical approach to $\chi \mathrm{SB}$ which is triggered and quantitatively determined by the increase of the gauge coupling.

\section{INSTANTON-MEDIATED INTERACTIONS, AXIAL ANOMALY AND THE FATE OF THE ETA BOSON}

Up to now, we have considered only that part of the model which has a global $U_{A}(1)$ symmetry corresponding to simultaneous axial phase rotations of the scalars and fermions. In QCD, this symmetry is anomalously broken by the presence of gauge-field configurations of nontrivial topology. For instance, instantons induce fermion interactions which break this symmetry. In an instanton-anti-instanton background, the $N_{\mathrm{f}}=1$ interaction is masslike and can be expressed as [19]

$$
\begin{aligned}
\mathcal{L}_{\mathrm{I}+\mathrm{A}}= & \int_{0}^{\bar{f}_{\mathrm{c}}\left(k, m_{\mathrm{f}}\right)} \frac{d \varrho}{\varrho^{5}} d_{0}^{N_{\mathrm{c}}}(\varrho) C_{\mathrm{E}}\left(N_{\mathrm{c}}\right)\left(2 \pi^{2} \varrho^{3}\right) \\
& \times\left(\frac{\alpha(1 / \rho)}{\alpha(\bar{\mu})}\right)^{-4 / b_{0}}\left(\bar{\psi}_{\mathrm{R}} \psi_{\mathrm{L}}-\bar{\psi}_{\mathrm{L}} \psi_{\mathrm{R}}\right), \\
d_{0}^{N_{\mathrm{c}}}(\varrho): & =\frac{4.6 e^{-1.68 N_{\mathrm{c}}}}{\pi^{2}\left(N_{\mathrm{c}}-1\right) !\left(N_{\mathrm{c}}-2\right) !}\left(\frac{2 \pi}{\alpha_{\mathrm{s}}(1 / \varrho)}\right)^{2 N_{\mathrm{c}}} e^{-2 \pi / \alpha_{\mathrm{s}}(1 / \varrho)},
\end{aligned}
$$

where $C_{\mathrm{E}}\left(N_{\mathrm{c}}\right)$ is a color factor that arises from averaging over all possible embeddings of $S U(2)$ into $S U\left(N_{\mathrm{c}}\right)$, e.g., $C_{\mathrm{E}}(2)=1, C_{\mathrm{E}}(3)=2 / 3$, and $\bar{\mu}=1 \mathrm{GeV}$ is the renormalization scale for the fermion fields. Note that we introduced an IR cutoff function $\bar{f}_{\mathrm{c}}\left(k, m_{\mathrm{f}}\right)$ in the upper bound of the instanton radius $\varrho$ integration. This function should cut off the contribution from all modes with momenta either below $k$ or the generated fermion mass $m_{\mathrm{f}}$, and thereby implements the renormalization group formulation of this interaction in a simple manner. The $\varrho$ integration is UV finite for $\varrho \rightarrow 0$ owing to asymptotic freedom, and the infrared $(\varrho \rightarrow \infty)$ is controlled by the cutoff $\bar{f}_{\mathrm{c}}$ and by the increase of the coupling.

In the following, we intend to include this interaction as it is, being an example for a $\mathrm{U}_{\mathrm{A}}(1)$ violating term. Contrary to standard instanton based models [20], we do not employ further information about, e.g., average instanton sizes and separations or other assumptions about the vacuum state of the gauge field. For this, we note that Eq. (36) already cor- responds to an integrated flow, $\mathcal{L}_{\mathrm{I}+\mathrm{A}}=\bar{m}_{\mathrm{I}+\mathrm{A}}\left(\bar{\psi}_{\mathrm{R}} \psi_{\mathrm{L}}-\bar{\psi}_{\mathrm{L}} \psi_{\mathrm{R}}\right)$, where the flow of the induced mass $\bar{m}_{\mathrm{I}+\mathrm{A}}$ is given by ${ }^{7}$

$$
\begin{aligned}
\partial_{t} \bar{m}_{\mathrm{I}+\mathrm{A}}= & 2 \pi^{2} Z_{\psi}\left[d_{0}^{N_{\mathrm{c}}}(\varrho) C_{\mathrm{E}}\left(N_{\mathrm{c}}\right)\right. \\
& \left.\times\left(\frac{\alpha(1 / \rho)}{\alpha(\bar{\mu})}\right)^{-4 / b_{0}}\right]_{\varrho=\bar{f}_{\mathrm{c}}\left(k, m_{\mathrm{f}}\right)} \partial_{t} \bar{f}_{\mathrm{c}}\left(k, m_{\mathrm{f}}\right),
\end{aligned}
$$

with the initial condition $\bar{m}_{\mathrm{I}+\mathrm{A}}(k=\Lambda \rightarrow \infty) \rightarrow 0$. For consistency, we also included here the fermion wave function renormalization $Z_{\psi}$, which was not taken into account in Eq. (36) as derived in [19]. Since $\bar{f}_{\mathrm{c}}$ has mass dimension -1 , an appropriate choice is given by

$$
\begin{aligned}
\bar{f}_{\mathrm{c}}\left(k, m_{\mathrm{f}}\right) & =\frac{1}{k} f_{\mathrm{c}}\left(\kappa h^{2}\right), \text { with } f_{\mathrm{c}}(0)=1, \\
\left.f_{\mathrm{c}}\left(\kappa h^{2}\right)\right|_{\kappa h^{2} \rightarrow \infty} & \rightarrow \frac{1}{\sqrt{\kappa h^{2}}},
\end{aligned}
$$

such that $\bar{f}_{\mathrm{c}}\left(0, m_{\mathrm{f}}\right)=1 / m_{\mathrm{f}}$. For our numerical solutions, we will use $f_{\mathrm{c}}(x)=(1+x)^{-1 / 2}$ for simplicity. With these definitions, we can rewrite Eq. (37) as

$$
\begin{aligned}
\partial_{t} \bar{m}_{\mathrm{I}+\mathrm{A}}= & -2 \pi^{2} Z_{\psi} \frac{k}{f_{\mathrm{c}}} d_{0}^{N_{\mathrm{c}}}\left(f_{\mathrm{c}} / k\right) C_{\mathrm{E}}\left(N_{\mathrm{c}}\right)\left(\frac{\alpha\left(k / f_{\mathrm{c}}\right)}{\alpha(\bar{\mu})}\right)^{-4 / b_{0}} \\
& \times\left(1+\frac{\left(-f_{\mathrm{c}}^{\prime}\right)}{f_{\mathrm{c}}} \partial_{t}\left(\kappa h^{2}\right)\right)
\end{aligned}
$$

where $f_{\mathrm{c}}=f_{\mathrm{c}}\left(\kappa h^{2}\right)$, and the prime denotes a derivative.

Now we could repeat the calculation of the flow equations of Sec. II including this fermion mass term in the propagator. In this way, however, we would induce a number of $\mathrm{U}_{\mathrm{A}}(1)$ noninvariant fermion-fermion and fermion-scalar couplings which complicate the calculation unnecessarily. Instead, we propose a generalization of the field transformation (23) which serves to translate the instanton-induced interaction into the scalar sector:

$$
\begin{aligned}
\partial_{t} \phi_{k}(q)= & -\left(\bar{\psi}_{\mathrm{L}} \psi_{\mathrm{R}}\right)(q) \partial_{t} \alpha_{k}(q)+\phi_{k}(q) \partial_{t} \beta_{k}(q)+\partial_{t} \gamma_{k} \\
& +\left(\phi_{k}^{*} \phi_{k}\right) \phi_{k} \partial_{t} \delta_{k}, \\
\partial_{t} \phi_{k}^{*}(q)= & \left(\bar{\psi}_{\mathrm{R}} \psi_{\mathrm{L}}\right)(-q) \partial_{t} \alpha_{k}(q)+\phi_{k}^{*}(q) \partial_{t} \beta_{k}(q)+\partial_{t} \gamma_{k} \\
& +\left(\phi_{k}^{*} \phi_{k}\right) \phi_{k}^{*} \partial_{t} \delta_{k},
\end{aligned}
$$

with additional a priori arbitrary functions $\gamma_{k}$ and $\delta_{k}$, whereas $\alpha_{k}$ and $\beta_{k}$ are those of Sec. II. The term $\sim \partial_{t} \gamma_{k}$ corresponds to a $U_{A}(1)$ violating shift of the scalar field

\footnotetext{
${ }^{7} \mathrm{~A}$ more rigorous treatment of anomalous $\mathrm{U}_{\mathrm{A}}(1)$ breaking within the flow equation formalism has been suggested in Ref. [21].
} 
which can compensate for the instanton-induced fermion mass. The flow of $\bar{m}_{\mathrm{I}+\mathrm{A}}$ is now given by

$$
\partial_{t} \bar{m}_{\mathrm{I}+\mathrm{A}}=\left.\partial_{t} \bar{m}_{\mathrm{I}+\mathrm{A}}\right|_{\phi_{k}}+\bar{h} \partial_{t} \gamma_{k}-\frac{1}{2} \bar{\nu} \partial_{t} \alpha_{k},
$$

where the second and third terms arise from the transformation of the Yukawa interaction and the last term in Eq. (6), respectively. Now we can determine $\gamma_{k}$ such that $\partial_{t} \bar{m}_{\mathrm{I}+\mathrm{A}}$ $=0$ holds on all scales. In this way, the instanton interaction does not affect $\bar{m}_{\mathrm{I}+\mathrm{A}}$ (which vanishes on all scales), but is translated into the scalar sector and contributes to the running of $\bar{\nu}$. In the point-like limit $\left(q^{2}=0\right)$, we find

$$
\partial_{t} \bar{\nu}=-2 \bar{m}^{2} \partial_{t} \gamma_{k}+\bar{\nu} \partial_{t} \beta_{k} .
$$

Introducing the dimensionless renormalized quantity

$$
\nu=\frac{\bar{\nu}}{Z_{\phi}^{1 / 2} k^{3}}, \quad \Rightarrow \quad \nu_{\mathrm{R}}=k^{3} \nu,
$$

where $\nu_{\mathrm{R}}$ denotes the renormalized (dimensionful) value, we finally arrive at

$$
\begin{aligned}
\partial_{t} \nu= & -\left(3-\frac{\eta_{\phi}}{2}\right) \nu-4 \pi^{2} \frac{\epsilon}{h} d_{0}^{N_{\mathrm{c}}}\left(f_{\mathrm{c}} / k\right) C_{\mathrm{E}}\left(N_{\mathrm{c}}\right) \\
& \times\left(\frac{\alpha\left(k / f_{\mathrm{c}}\right)}{\alpha(\bar{\mu})}\right)^{-4 / b_{0}} \frac{1}{f_{\mathrm{c}}}\left(1+\frac{\left(-f_{\mathrm{c}}^{\prime}\right)}{f_{\mathrm{c}}} \partial_{t}\left(\kappa h^{2}\right)\right) \\
& +\frac{\nu}{h^{2}}\left[1+(1+\epsilon)^{2} Q_{\sigma}\right]\left(\beta_{\bar{\lambda}_{\sigma}}^{g^{4}} g^{4}+\beta_{\bar{\lambda}_{\sigma}}^{h^{4}} h^{4}\right),
\end{aligned}
$$

which describes the running of the axial anomaly in the instanton approximation.

The shift $\sim \partial_{t} \gamma_{k}$ induces another $\mathrm{U}_{\mathrm{A}}(1)$ violating term $\left(\phi^{*} \phi\right)\left(\phi^{*}+\phi\right)$ via the transformation of the $\lambda_{\phi}\left(\phi^{*} \phi\right)^{2}$ term. This can be cancelled by an appropriate choice of the last transformation function $\delta_{k}$ in Eq. (40), which has to satisfy

$$
\bar{\lambda}_{\phi} \partial_{t} \gamma_{k}-\frac{1}{2} \bar{\nu} \partial_{t} \delta_{k}=0
$$

Finally, the terms $\sim \delta_{k}$ in Eq. (40) influence the running of $\lambda_{\phi}$ via the transformation of the scalar mass term. The modified flow equation for $\lambda_{\phi}$ reads

$$
\begin{aligned}
\partial_{t} \lambda_{\phi}= & \left.\partial_{t} \lambda_{\phi}\right|_{\phi_{k}}+4 \frac{\lambda_{\phi}}{h^{2}}\left(1+2 \epsilon+(1+\epsilon)^{2} Q_{\sigma}\right)\left(\beta_{\lambda_{\sigma}}^{g^{4}} g^{4}+\beta_{\lambda_{\sigma}}^{h^{4}} h^{4}\right) \\
& +16 \pi^{2} \frac{\epsilon \lambda_{\phi}}{\nu h} d_{0}^{N_{\mathrm{c}}}\left(f_{\mathrm{c}} / k\right) C_{\mathrm{E}}\left(N_{\mathrm{c}}\right)\left(\frac{\alpha\left(k / f_{\mathrm{c}}\right)}{\alpha(\bar{\mu})}\right)^{-4 / b_{0}} \\
& \times \frac{1}{f_{\mathrm{c}}}\left(1+\frac{\left(-f_{\mathrm{c}}^{\prime}\right)}{f_{\mathrm{c}}} \partial_{t}\left(\kappa h^{2}\right)\right) .
\end{aligned}
$$

These equations are valid in the symmetric regime with similar equations for the $\chi \mathrm{SB}$ regime displayed in Appendix C.

Strictly speaking, the system is never in the symmetric regime, since chiral symmetry is always broken implicitly by a nonzero $\nu$ term which induces a nonzero VEV $\sigma_{0}$ for the scalar field. For instance, rotating the VEV into the real component, $\phi=\sigma_{0}=\phi^{*}, \sigma_{0}=\sqrt{\rho_{0}}$, the location of the minimum obeys

$$
0=U^{\prime}\left(\rho_{0}\right)=\bar{m}^{2}+\bar{\lambda}_{\phi} \rho_{0}-\frac{\bar{\nu}}{2 \sqrt{\rho_{0}}} \Rightarrow 0=\epsilon+\kappa \lambda_{\phi}-\frac{1}{2} \frac{\nu}{\sqrt{\kappa}} .
$$

Obviously, $\kappa=0$ is not allowed if $\nu \neq 0$, owing to the linear term in $\phi$ in Eq. (6). The running of the minimum can be inferred from

$$
\begin{gathered}
0=\left.\partial_{t} U^{\prime}\left(\rho_{0}\right)\right|_{\rho}=U^{\prime \prime}\left(\rho_{0}\right) \partial_{t} \rho_{0}+\left.\partial_{t} U^{\prime}\left(\rho_{0}\right)\right|_{\rho_{0}} \\
\Rightarrow \partial_{t} \rho_{0}=-\left.\frac{1}{\bar{\lambda}_{\phi}+\frac{\bar{\nu}}{4} \rho_{0}^{-3 / 2}} \partial_{t} U^{\prime}\left(\rho_{0}\right)\right|_{\rho_{0}} .
\end{gathered}
$$

Since the instanton-induced terms are exponentially small for the major part of the flow, the minimum of the potential is actually very close to zero, and the equations for the symmetric regime of Sec. II can be used up to tiny corrections. The solution of the flow equations is numerically difficult with an exponentially small $\kappa$ in the broken regime. Therefore, we decide to solve the flow equations for large enough $k$ in the symmetric-regime formulation. In this regime, $\nu$ evolves according to Eq. (44) with only a subdominant coupling to the other flow equations via Eq. (46). Then we switch to the broken-regime description at that scale where the instanton-induced fermion mass $m_{\mathrm{f}}$ is of the order of a few $\mathrm{MeV}$; this procedure induces an error only at the permille level and turns out to be insensitive to the details of the switching scale.

We have analyzed the flow equations including the instanton-mediated interaction numerically and used the reference set of initial conditions as defined in Sec. IV [see Eq. (33)] for a direct comparison. As expected, most properties of the system are unaffected by the instantons, while the system is governed by the bound-state fixed point. Here the instanton-induced effects are exponentially suppressed, since the coupling is small. In particular, the running of the scalar mass $\epsilon$ and the Yukawa coupling are identical to the ones displayed in Fig. 3, and the universality properties discussed in Sec. IV remain unaffected.

The renormalized axial anomaly $\nu_{\mathrm{R}}$ is plotted in Fig. 6 (left panel). It remains exponentially small for a large part of the flow and becomes of order $(\mathrm{GeV})^{3}$ and larger only in the strong-gauge-coupling regime. Here, however, it contributes strongly to the VEV of the scalar field and consequently to the constituent quark mass which leads to the decoupling of the fermions.

We observe a rather smooth onset of fermion-mass generation. Furthermore, the constituent quark mass is strongly 

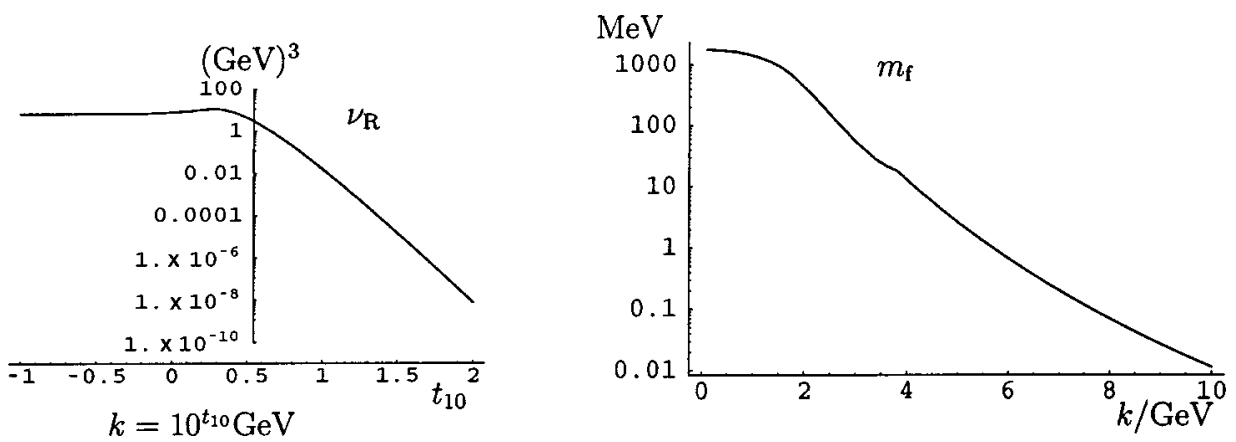

FIG. 6. Left panel: axial anomaly $\nu_{\mathrm{R}}$ in the vicinity of the scale of fermion decoupling. Right panel: instanton-induced fermion mass. Both plots refer to the reference set (33) and the particular choice for the running of the gauge coupling according to Eq. (35) with $\alpha_{*}$ $=2.5$. A comparison with Fig. 4 shows that the fermion mass is dominated by instanton effects.

enhanced by the instanton interactions. For the reference set, we find $m_{\mathrm{f}}=1765 \mathrm{MeV}$ in the infrared limit $k \rightarrow 0$. Again, this number depends strongly on the precise choice of the running gauge coupling in the infrared, and a number of other possibilities including instanton effects is listed in Appendix B.

Let us finally discuss the fate of the "would-be" Goldstone boson, which we may call the eta boson in the style of real QCD. Neglecting the axial anomaly, this boson arises from spontaneous breakdown of the global $\mathrm{U}_{\mathrm{A}}(1)$ as a true massless Goldstone boson; its effects on the scalar sector even after $\chi \mathrm{SB}$ are visible in the logarithmic running of the scalar $\phi^{4}$ coupling $\lambda_{\phi}$ as can be seen in Fig. 5. The $\mathrm{U}_{\mathrm{A}}(1)$ anomaly, however, generates a mass of the eta boson. In the present formulation, the $\mathrm{U}_{\mathrm{A}}(1)$ anomaly occurs as the $\bar{\nu}$ term in the scalar potential (6). Its contribution to the renormalized eta mass can be computed as

$$
m_{\eta}^{2}=\frac{\nu_{\mathrm{R}}}{2 \sigma_{\mathrm{R}}} .
$$

Within the above-given framework of instanton-mediated interaction, we find for the eta boson mass in the QCD universality class a value of $m_{\eta} \simeq 4440 \mathrm{MeV}$. Of course, this value also strongly depends on the choice of the running of the gauge coupling and should be used only for comparison with other masses computed for the same running gauge coupling. In particular, we find roughly the ratio $m_{\eta} / m_{\mathrm{f}} \simeq 3$. This scenario giving rise to a heavy mass of a would-be Goldstone boson is familiar from three-flavor QCD.

By contrast, the fate of the eta boson is more spectacular if we go beyond the border of the QCD domain to that of $\mathrm{P} \chi \mathrm{SB}$, corresponding to a choice of $\bar{m}_{\Lambda}^{2}<\bar{m}_{\mathrm{c}}^{2}$ in Fig. 1 or $\tilde{\epsilon}_{\Lambda}<\tilde{\epsilon}_{1}^{*}$ in Fig. 2. Here, the VEV of the scalar field is generically of the order of the cutoff $\Lambda=10^{15} \mathrm{GeV}$. At the same time, the fermions rapidly become massive and decouple from the flow only a little below $\Lambda$. As a consequence, instanton contributions or other long-distance topological properties have little effect on the fermion sector and thus the axial anomaly exerts hardly any influence on the scalars. As a result, the contributions to the eta mass are strongly suppressed-powerlike in the denominator and exponentially in the numerator. For instance, for the set of initial param- eters corresponding to Fig. 1 (right panel) with $\bar{m}_{\Lambda}^{2}$ slightly below $\bar{m}_{\mathrm{c}}^{2}$, we find an extremely small eta mass, $m_{\eta} \simeq 2$ $\times 10^{-30} \mathrm{eV}$. For smaller $\bar{m}_{\Lambda}^{2}$, the eta mass decreases even further, and larger eta masses require a tremendous finetuning of $\bar{m}_{\Lambda}^{2}$ close to $\bar{m}_{\mathrm{c}}^{2}$.

In this scenario beyond the QCD universality class, we have thus found a mechanism to generate extremely small masses without any fine tuning. From another perspective, this mechanism exploits the fundamentally different RG properties of scalars and chiral gauge theories. For systems in the universality class of $\mathrm{P} \chi \mathrm{SB}$, the $\chi \mathrm{SB}$ scale of the scalar sector is generically of the order of the UV scale, whereas the nonperturbative scale of the gauge sector can be much smaller. Now the mass of the would-be Goldstone boson is generated by the nonperturbative sector of the gauge theory which is exponentially suppressed at the UV scale. This interplay finally leads to the generation of the extremely small mass.

\section{CONCLUSIONS}

In this work, we studied a class of theories involving oneflavor massless QCD and a chiral color-singlet scalar field. Our model is parametrized by the gauge coupling and a number of scalar couplings. In this framework, we identified the QCD universality class of theories which share the same physics at low energies, namely spontaneous breaking of chiral symmetry triggered by the strongly interacting gauge sector at the QCD scale. As a remarkable result, the QCD universality class contains theories with fundamental scalars where the microscopic scalar potential has its minimum at nonzero field $\left[\bar{m}_{\Lambda}^{2}>\bar{m}_{\mathrm{c}}^{2} \sim-\mathcal{O}\left(\Lambda^{2}\right)\right]$. For these the theories, the scalar fluctuations drive the system first into the symmetric regime with a large positive scalar mass, and the remaining flow is governed by the QCD sector. We checked explicitly that this is in accord with perturbative expectations for weak couplings (cf. Fig. 1, right panel).

The mechanism that establishes QCD universality is the occurrence of an infrared attractive bound-state fixed point in the scalar couplings which persists over a wide range of scales as long as the gauge coupling is weak. At this fixed point, the scalar field exhibits quark-antiquark bound-state behavior and the RG running of the scalar couplings is gov- 
erned by the RG behavior of QCD. All memory of the scalar initial conditions is lost by the system. As a remarkable consequence, the scalar mass is not a relevant operator at this fixed point. For increasing gauge coupling, the bound-state fixed point is destabilized and the system runs towards the $\chi \mathrm{SB}$ regime. Here the role of the scalar field changes and it can characterize (quark) condensates and (mesonic) excitations on top of the condensate. At strong coupling, the simple overall picture of $\chi \mathrm{SB}$ arising from our truncation can, of course, be modified quantitatively as well as qualitatively by the influence of higher-order operators. In particular, mixed nonminimal fermion-gluon and scalar-gluon operators might add new features to $\chi \mathrm{SB}$ by providing a coupling to the nontrivial gluonic vacuum structure.

Beyond the QCD universality class, we find the class of theories exhibiting perturbative spontaneous chiral symmetry breaking $(\mathrm{P} \chi \mathrm{SB})$. In this class, the system is mainly driven by the scalar sector, and IR properties such as condensates and generated fermion masses depend strongly on the initial scalar parameters. The gauge sector exerts hardly any influence on the fermions in this class unless the scalar parameters are fine tuned to a high precision. In the deep IR, pure gluodynamics without dynamical quarks remains. The flow of the scalar couplings is never in the attractive domain of the bound-state fixed point, but is governed by a fundamental-particle fixed point. Small deviations from this fixed point have an infrared unstable component which corresponds to the RG relevant scalar-mass operator.

In both universality classes, we found interesting implications. Our setup of the QCD universality class admits a resolution of an old puzzle: whereas QCD has no fine-tuning problem and is completely determined by fixing the coupling at a certain scale, low-energy QCD models based on NJLtype fermion self-interactions depend strongly on additional parameters such as an intrinsic UV cutoff. In the context of partial bosonization, this cutoff dependence corresponds to a strong dependence of IR observables on the bosonization scale (or the value of the scalar mass at this scale). In our approach with scale-dependent field transformations, partial bosonization occurs at all scales, and no artificial dependence on unphysical scales is introduced. In our truncation, QCD flows continuously from a high scale with quarks and gluons as the relevant degrees of freedom to intermediate scales with quarks, gluons and quark bound states and further to low scales with constituent quarks, condensates and mesons.

In the $\mathrm{P} \chi \mathrm{SB}$ universality class with one fermion flavor, we identified a natural mechanism for the generation of extremely small scalar masses without fine tuning. The mechanism exploits the fact that the spontaneous breaking of the $\mathrm{U}_{\mathrm{A}}$ (1) symmetry would lead to an exactly massless Goldstone boson in the absence of gauge interactions. The axial anomaly in the gauge sector then endows a small mass to this boson. Owing to the highly different RG behavior of the scalar and the gauge sector, the scale of $\mathrm{P} \chi \mathrm{SB}$ differs generically from the scale of nonperturbative gauge effects by many orders of magnitude. This leads to an exponential suppression of the influence of the axial anomaly and thus to an exponentially small but nonzero scalar mass.

For theories with a fundamental scalar, the question arises as to whether our technique of fermion-boson translation is capable of describing all possible mesonic degrees of freedom. Let us first look at two extreme situations. For a large negative renormalized scalar mass term, perturbation theory applies: there is a fundamental scalar, and separately propagating meson states may not exist, similar to a very heavy top quark. For a positive renormalized mass term, the fundamental scalar decouples from the low-energy sector in perturbation theory. The low-energy sector then is QCD without scalars, as in our picture. The transition is less obvious: in the region where the fundamental scalar mass would perturbatively be of the order of the strong interaction scale, there is a strong mixing between operators corresponding to fundamental and composite scalars.

In principle, in a situation with mixing the propagator in the scalar sector may have one or several pole-like structures that can be associated with particle excitations. Our truncation cannot fully resolve this issue, since, by construction, it follows the flow of only one pole in the propagator. Our investigation shows the consistency of a picture with only one pole. If the true physical situation had two poles our truncation would follow the flow of the lowest mass. We see, however, no indication that a second pole actually exists. Nevertheless, it seems worthwhile to discuss the possible implications of a second scalar "pole" for the issue of universality classes. First of all, for $\bar{m}_{\Lambda}^{2}$ larger than but not in the immediate vicinity of $\bar{m}_{\mathrm{c}}^{2}$, a "second pole" could correspond only to an additional heavy scalar particle. This would decay at a high rate into the QCD mesons, since no quantum numbers forbid such a decay. (One expects at most a resonance rather than a true pole.) Furthermore, effects from the exchange of such a heavy scalar resonance would be suppressed by inverse powers of the mass and therefore play no role for the low-energy theory. This is what one usually understands by "QCD universality class" (a notion that is not thought to resolve the detailed short-distance physics).

This issue becomes more interesting when $\bar{m}_{\Lambda}^{2}$ is finetuned to the immediated vicinity of $\bar{m}_{\mathrm{c}}^{2}$. In this case, we approach the boundary of the QCD universality class. We emphasize that this boundary is not uniquely defined in terms of the symmetries and particles characteristic for the QCD universality class. Considering the QCD universality class from the viewpoint of a larger space of models or parameters, the spectrum of excitations that are relevant at the boundary can depend on the direction in parameter space from which the QCD universality class is approached. Different directions may yields a different "number of poles" in the boundary region. For this reason, a future more detailed investigation of this issue would be quite interesting.

We stress that all of our main conclusions can be drawn from a mere perturbative knowledge of the gauge sector which is well under control. In a broader sense, the pure QCD sector in our work can be regarded as a particular example for possible other (nonperturbatively) renormalizable theories leading to fermionic self-interactions in scalar channels.

Let us finally discuss our findings from a different perspective, concentrating on the scalar sector. Scalar fields are 
known to lead to profound problems in quantum field theory for two reasons: triviality and (un-)naturalness. Triviality tells us that an interacting scalar theory requires a UV cutoff which cannot be removed without switching off the interaction. Therefore, whenever we see a scalar quantum field at some low scale, we know that there must be new physics at a higher scale. The problem of naturalness tells us that it is difficult to achieve a large separation of scales for models with interacting scalar fields without fine tuning.

Our formulation has the potential to solve both problems. A first example can be given within the QCD universality class. Although from a QCD perspective, the scalar field could be regarded as purely auxiliary, nothing prevents us from considering it as fundamental, since the concepts of compositeness and fundamentality are interchangeable from the viewpoint of our flow equation with field transformations. We showed in detail that "standard" QCD at low energies is indistinguishable from QCD with a fundamental scalar, as long as the latter system is in the QCD universality class. In this way, we can circumvent triviality by starting in the UV from a scalar field theory without self-interaction and Yukawa coupling for which the continuum limit can be taken trivially. The scalar interactions are induced by quantum fluctuations. In this construction, the system is always in the QCD universality class, and therefore inherits the number of relevant and marginal operators form QCD. In particular, the scalar mass term is not a relevant operator, so that no naturalness or fine-tuning problems arise in and from the scalar sector. Alternatively, we could also follow the bound-state fixed point to $k \rightarrow \infty$, where it presumably becomes an exact fixed point even beyond our truncation. (The $\beta$ function for the running gauge coupling vanishes, owing to asymptotic freedom in this limit.)

Perhaps more interesting is a second possibility in the $\mathrm{P} \chi \mathrm{SB}$ class. Let us consider for $k \rightarrow \infty$ a scalar model with $Z_{\phi} \rightarrow 0$ and $\lambda_{\phi} \rightarrow 0$ and $\bar{m}^{2}$ and $\bar{h}$ chosen such that $\tilde{\epsilon}$ corresponds to the fixed point $\tilde{\epsilon}_{1}^{*}$. This model has an alternative interpretation as a model with four-fermion interactions (and no scalar field). Both the gauge coupling and the critical four fermion coupling

$$
\bar{\lambda}_{\sigma}^{*}=\frac{1}{2 \tilde{\epsilon}_{1}^{*} k^{2}}
$$

vanish for $k \rightarrow \infty$. If this fixed point persists beyond our truncation, it defines a nonperturbatively renormalizable theory [22]. For lower $k$ a nonzero $\lambda_{\phi}$ is generated by the flow and we end up with a theory that effectively looks like a model with an interacting fundamental scalar field. This scalar field can give mass to the quarks by $\mathrm{P} \chi \mathrm{SB}$ independently of the strong interactions, in analogy to the Higgs scalar. The triviality problem could be solved in this case-but not the naturalness problem, since we expect a relevant parameter corresponding to the scalar mass term.

This discussion sheds new light on the continuous transition between the $\mathrm{P} \chi \mathrm{SB}$ and $\mathrm{QCD}$ universality classes. In the language of statistical physics, it can be considered as a type of crossover between the "fundamental fixed point" $\tilde{\epsilon}_{1}^{*}$ and the "bound-state fixed point" $\tilde{\epsilon}_{2}^{*}$. As a particularity, the gauge coupling is a marginal parameter for both fixed points. The scale where it becomes strong sets the lowest possible scale for the effective fermion masses.

Quite generally, the existence of a bound-state-like fixed point leads to a mechanism with a naturally small scalar mass. In a sense, this is a realization of earlier ideas of a large anomalous mass dimension for the scalar field or "selforganized criticality" [23]. It would be interesting to know if a similar mechanism could contribute to an understanding of electroweak symmetry breaking which occurs at a characteristic scale hundreds of times bigger as compared to QCD.

\section{ACKNOWLEDGMENTS}

The authors thank J. Jaeckel for valuable discussions. H.G. acknowledges financial support by the Deutsche Forschungsgemeinschaft under Contract No. Gi 328/1-1.

\section{APPENDIX A: THRESHOLD FUNCTIONS}

The regularization scheme dependence induced by the cutoff function $R_{k}$ is carried by the threshold functions $l$ and $m$. Let us represent the cutoff functions in the scalar, fermion and gauge sector by

$$
\begin{gathered}
R_{k}^{\phi}\left(q^{2}\right)=Z_{\phi} q^{2} r(y), \quad R_{k}^{\psi}(q)=-Z_{\psi} q / r_{\mathrm{F}}(y), \\
\left(R_{k}^{A}(q)\right)_{\mu \nu}=Z_{\mathrm{F}} q^{2} r(y)\left[g_{\mu \nu}-\left(1-\frac{1}{\xi}\right) \frac{q_{\mu} q_{\nu}}{q^{2}}\right],
\end{gathered}
$$

where $y=q^{2} / k^{2}$, and $r$ and $r_{\mathrm{F}}$ denote dimensionless cutoff shape functions. Furthermore, it is useful to introduce the inverse average propagators $P(x)=x\left[1+r\left(x / k^{2}\right)\right]$ and $P_{\mathrm{F}}(x)=x\left[1+r_{\mathrm{F}}\left(x / k^{2}\right)\right]^{2}$, where $x=q^{2}$.

Most of the threshold functions given above are defined in Appendix A of Ref. [4]. The ones which cannot be found therein are marked with a tilde. These can be defined as follows:

$$
\begin{aligned}
\tilde{m}_{1,1}^{(\mathrm{FB}), d}\left(w_{\mathrm{F}}, w_{\mathrm{B}} ; \eta_{\psi} ; \eta_{\phi}\right) \\
\quad=-\frac{1}{2} k^{4-d} \int_{0}^{\infty} d x x^{d / 2-1} \widetilde{\partial}_{t}\left[\frac{1+r_{\mathrm{F}}\left(x / k^{2}\right)}{P_{\mathrm{F}}(x)+k^{2} w_{\mathrm{F}}} \frac{1}{P(x)+k^{2} w_{\mathrm{B}}}\right],
\end{aligned}
$$

$$
\begin{aligned}
\widetilde{l}_{1,2}^{(\mathrm{FB}), d}\left(w_{\mathrm{F}}, w_{\mathrm{B}} ; \eta_{\psi}, \eta_{\mathrm{B}}\right) \\
=-\frac{1}{2} k^{6-d} \int_{0}^{\infty} d x x^{d / 2-1} \widetilde{\partial}_{t} \\
\quad \times\left[\frac{P_{\mathrm{F}}(x)}{\left(P_{\mathrm{F}}(x)+k^{2} w_{\mathrm{F}}\right)^{2}} \frac{1}{\left(P(x)+k^{2} w_{\mathrm{B}}\right)^{2}}\right],
\end{aligned}
$$




$$
\begin{aligned}
\widetilde{l}_{1,1,1}^{\mathrm{FBB}), d}\left(w_{\mathrm{F}}, w_{\mathrm{B} 1}, w_{\mathrm{B} 2} ; \eta_{\psi}, \eta_{\mathrm{B}}\right) \\
=-\frac{1}{2} k^{6-d} \int_{0}^{\infty} d x x^{d / 2-1} \widetilde{\partial}_{t} \\
\quad \times\left[\frac{P_{\mathrm{F}}(x)}{\left(P_{\mathrm{F}}(x)+k^{2} w_{\mathrm{F}}\right)^{2}} \frac{1}{P(x)+k^{2} w_{\mathrm{B} 1}} \frac{1}{P(x)+k^{2} w_{\mathrm{B} 2}}\right],
\end{aligned}
$$

where $\eta_{\mathrm{B}}$ denotes one of the anomalous dimensions of the bosonic propagators under consideration, $\eta_{\phi}$ or $\eta_{\mathrm{F}}$ in our case. The derivative $\widetilde{\partial}_{t}$ acts on the $k$ dependence of the cutoff function only (for an explicit representation of $\widetilde{\partial}_{t}$; see Ref. [4]). Some relations among the threshold functions are given by

$$
\begin{aligned}
& \widetilde{l}_{1,1,1}^{(\mathrm{FBB}), d}\left(w_{\mathrm{F}}, w_{\mathrm{B}}, w_{\mathrm{B}} ; \eta_{\psi}, \eta_{\mathrm{B}}\right) \equiv \widetilde{l}_{1,2}^{(\mathrm{FB}), d}\left(w_{\mathrm{F}}, w_{\mathrm{B}} ; \eta_{\psi}, \eta_{\mathrm{B}}\right), \\
& \widetilde{l}_{1,2}^{(\mathrm{FB}), d}\left(w_{\mathrm{F}}=0, w_{\mathrm{B}} ; \eta_{\psi}, \eta_{\mathrm{B}}\right)=l_{1,2}^{(\mathrm{FB}), d}\left(w_{\mathrm{F}}=0, w_{\mathrm{B}} ; \eta_{\psi}, \eta_{\mathrm{B}}\right) .
\end{aligned}
$$

For our numerical computations, we use the linear cutoff functions proposed in Ref. [16] $\left(y=q^{2} / k^{2}\right)$,

$$
r(y)=\left(\frac{1}{y}-1\right) \theta(1-y), \quad r_{\mathrm{F}}(y)=\left(\frac{1}{\sqrt{y}}-1\right) \theta(1-y),
$$

for which all integrals listed above can be performed analytically, yielding in the present context:

$$
\begin{aligned}
& \tilde{m}_{1,1}^{(\mathrm{FB}), d}\left(w_{\mathrm{F}}, 0 ; \eta_{\psi}, \eta_{F}\right) \\
& =\frac{2}{d-1} \frac{1}{1+w_{\mathrm{F}}}\left[\frac{1}{2}\left(1+\frac{d}{2} \eta_{\psi}\right)-\frac{\eta_{F}}{d+1}+\frac{\left(1-\frac{d}{2} \eta_{\psi}\right)}{1+w_{\mathrm{F}}}\right], \\
& \widetilde{l}_{1,2}^{\mathrm{FB}), d}\left(w_{\mathrm{F}}, 0 ; \eta_{\psi}, \eta_{F}\right)=\frac{2}{d} \frac{1}{\left(1+w_{\mathrm{F}}\right)^{2}}\left[\left(1-\frac{2 \eta_{F}}{d+2}+\frac{\eta_{\psi}}{d+1}\right)\right. \\
& \left.\quad+\frac{2}{1+w_{\mathrm{F}}}\left(1-\frac{\eta_{\psi}}{d+1}\right)\right], \\
& \quad \frac{2}{d} \frac{(\mathrm{A} 7}{\left(1+w_{\mathrm{F}}\right)^{2}\left(1+w_{1}\right)\left(1+w_{2}\right)}\left[\left(\frac{1}{1+w_{1}}+\frac{1}{1+w_{2}}\right)\right. \\
& \left.\quad \times\left(1-\frac{\eta_{\phi}}{d+2}\right)+\left(\frac{2}{1+w_{\mathrm{F}}}-1\right)\left(1-\frac{\eta_{\psi}}{d+1}\right)\right] .
\end{aligned}
$$

The representations of all other threshold functions for the linear cutoff can be looked up in Ref. [13].

\section{APPENDIX B: NONPERTURBATIVE RUNNING OF THE GAUGE COUPLING}

The infrared quantities serving as "physical observables" in the present work, such as the constituent quark mass or the eta boson mass, depend on the way we model the effective gauge coupling in the nonperturbative domain in our truncation. In order to gain more insight into this dependence, we study different gauge coupling $\beta$ functions proposed in the literature in this appendix. Here we focus on theories within the QCD universality class which are sensitive to the infrared physics of the gauge sector.

In Sec. IV, we used a $\beta$ function with accurate two-loop behavior and an IR fixed point at $\alpha_{*}=2.5$. We denote this $\beta$ function defined in Eq. (35) serving as a reference as $\beta_{\text {Ref }}$ in the following. Such $\beta$ functions with a fixed point of the gauge coupling in the infrared have a long tradition in the literature and have frequently been discussed from a phenomenological viewpoint [24]. Furthermore, some theoretical evidence for the existence of such a fixed point has been collected in certain nonperturbative approximation schemes. However, due to the lack of a unique nonperturbative definition of the gauge coupling and due to an inherent regularization scheme dependence of the $\beta$ function, a comparison of different theoretical approaches and a connection to phenomenology is difficult to make. Here we take a pragmatic point of view and use the various running couplings as effective ones which are implicitly defined by their use in our approach.

Recently, an actual nonperturbative computation of the running coupling has been set up in the framework of truncated Schwinger-Dyson equations in Landau gauge [25], revealing an infrared fixed point; these results also receive some support from lattice calculations [26]. For our purposes, we use the representation given in Ref. [27] for the running coupling,

$$
g_{\mathrm{SDE}}^{2}(x)=\frac{4 \pi \alpha_{*, \mathrm{SDE}}}{\ln \left(e+a_{1} x^{a_{2}}+c_{1} x^{c_{2}}\right)} \quad \text { where } \quad \alpha_{*, \mathrm{SDE}}=2.972
$$

and $a_{1}=5.292 \mathrm{GeV}^{-2 a_{2}}, a_{2}=2.324, c_{1}=0.034 \mathrm{GeV}^{-2 c_{2}}$, and $c_{2}=3.169$. This coupling is also normalized to the standard value at the $Z$ mass, and we identify $x=k^{2} /(\mathrm{GeV})^{2}$. The $\beta$ function is given by $\beta_{\mathrm{SDE}}=\partial_{t} g_{\mathrm{SDE}}^{2}$.

As a second example, we use the running coupling arising from a scheme called "analytic perturbation theory" [28] that has been devised for enforcing analyticity properties of the coupling in the timelike and spacelike (Euclidean) region. For our numerical routine, we use the approximate (but two-loop accurate) representation

$$
\begin{aligned}
g_{\mathrm{APT}}^{2}(x) & =\frac{(4 \pi)^{2}}{b_{0}}\left(\frac{1}{l_{2}(x)}+\frac{1}{1-\exp \left[l_{2}(x)\right]}\right), \\
l_{2}(x) & =\ln x+\frac{b_{1}}{b_{0}^{2}} \ln \sqrt{\ln ^{2} x+4 \pi^{2}}
\end{aligned}
$$


where we identify $x=k^{2} /(1349 \mathrm{MeV})^{2}$, so that this coupling is also normalized at the $Z$ mass. In the infrared $k \rightarrow 0$, the coupling tends to the fixed point $\alpha_{*, \mathrm{APT}}=4 \pi / b_{0} \simeq 1.22$ for $N_{\mathrm{c}}=3$ and $N_{\mathrm{f}}=1$.

As a third example, we use a calculation of the running coupling based on a truncated flow equation that also revealed an infrared fixed point $\alpha_{*, \mathrm{FE}}$ [29]. The corresponding $\beta_{\mathrm{FE}}$ function was obtained as an extensive multiple integral which we will not display here. Since this result holds for pure gauge theory, we incorporate one quark flavor in a "quenched" approximation by adding the fermionic part of the two-loop $\beta$ function to the pure gauge result. This leads to an infrared fixed-point value of $\alpha_{*, \mathrm{FE}} \simeq 3.43 \pm 0.01$, where the theoretical error arises from an incompletely resolved color structure in Ref. [29]. We would like to point out that the definition of the running coupling used in Ref. [29] agrees with the one of the present work.

As a simple example for a running coupling which does not tend to an infrared fixed point, we employ a class of $\beta$ functions that correspond to anomalous dimensions of the gauge field $\eta_{F}$ which become constant for $k \rightarrow 0$. This is realized by the choice

$$
\begin{aligned}
\beta_{\eta_{*}}= & -2\left(b_{0} \frac{g^{4}}{16 \pi^{2}}+b_{1} \frac{g^{6}}{\left(16 \pi^{2}\right)^{2}}\right) \\
& \times\left[1-\exp \left(-\frac{\left(16 \pi^{2}\right)^{2}}{2 b_{1} g^{4}}\left(-\eta_{*}\right)\right)\right],
\end{aligned}
$$

so that in fact $\eta_{F}=\beta_{\eta_{*}} / g^{2} \rightarrow \eta_{*}$ for $k \rightarrow 0$. For negative $\eta_{*}$, the running coupling increases $\sim(1 / k)^{\left|\eta_{*}\right|}$ for $k \rightarrow 0$. As explicit examples, we choose $\eta_{*}=-0.1$ and $\eta_{*}=-0.5$ for the numerical analysis.

The results of the numerical integration of the flow equations are collected in Table I. For the various $\beta$ functions denoted in the first column, we listed the transition scale $k_{\chi}$ SB into the $\chi \mathrm{SB}$ regime and the generated fermion mass $m_{\mathrm{f}}$ in the next two columns. These results refer to calculations without axial anomaly and instanton-mediated interactions, similarly to Sec. IV. In the last two columns the fermion mass with instanton contribution and the mass of the eta boson are given.

Obviously, the quantities $k_{\chi}$ SB and $m_{\mathrm{f}}$ in the calculation without axial anomaly are roughly correlated. Furthermore, $\beta_{\mathrm{APT}}$ and $\beta_{\eta_{*}=-0.1}$ lead to small values for $k_{\chi \mathrm{SB}}$ and $m_{\mathrm{f}}$, since both approach larger values of the coupling only very slowly. The fermion and eta boson masses including the axial anomaly are not strictly correlated with the former quantities. The running of the gauge coupling enters these quantities over a wider range of scales, since sizable instanton contributions can already arise while the bound-state fixed point is still present.

Nevertheless, our main observation is that the overall qualitative picture of the approach to $\chi \mathrm{SB}$, even in the nonperturbative domain, is rather independent of the details of
TABLE I. Characteristic masses $m_{\mathrm{f}}$ and $m_{\eta}$ for various nonperturbative $\beta$ functions for the strong gauge coupling. The main uncertainty concerns the overall scale, whereas the ratio $m_{\eta} / m_{\mathrm{f}}$ is relatively robust. We also show the scale of transition to $\chi \mathrm{SB}$ and the fermion mass $\tilde{m}_{\mathrm{f}}$ in the absence of instanton effects.

\begin{tabular}{lccccc}
\hline \hline$\beta$ function & $m_{\mathrm{f}} / \mathrm{MeV}$ & $m_{\eta} / \mathrm{MeV}$ & $m_{\eta} / m_{\mathrm{f}}$ & $k_{\chi \mathrm{SB}} / \mathrm{MeV}$ & $\tilde{m}_{\mathrm{f}} / \mathrm{MeV}$ \\
\hline$\beta_{\mathrm{Ref}}$ & 1765 & 4438 & 2.5 & 423 & 371 \\
$\beta_{\mathrm{SDE}}$ & 1777 & 4226 & 2.4 & 457 & 427 \\
$\beta_{\mathrm{APT}}$ & 563 & 1990 & 3.5 & 4 & 3 \\
$\beta_{\mathrm{FE}}$ & $903 \pm 2$ & $2117 \pm 1$ & 2.3 & $243 \pm 2$ & $241 \pm 3$ \\
$\beta_{\eta_{*}=-0.1}$ & 513 & 1793 & 3.5 & 11 & 8 \\
$\beta_{\eta_{*}=-0.5}$ & 1946 & 4534 & 2.3 & 394 & 395 \\
\hline \hline
\end{tabular}

the gauge sector in our truncation. On the one hand, a strong gauge coupling $g^{2}>g_{\mathrm{D}}^{2}$ is all that is needed to trigger $\chi \mathrm{SB}$; on the other hand, fermion decoupling cuts off any strong influence of the running coupling in the deep infrared. Quantitative results, of course, depend strongly on the flow of the coupling between the scale at which $g^{2}=g_{\mathrm{D}}^{2}$ and the scale of fermion decoupling. This mainly concerns the overall scale, whereas mass ratios like $m_{\eta} / m_{\mathrm{f}}$ turn out to be more robust.

\section{APPENDIX C: FLOW EQUATIONS WITH AXIAL ANOMALY IN THE BROKEN REGIME}

Here we collect the flow equations for the various couplings in the broken regime, including the contributions arising from the axial anomaly. Let us begin with the scalar and fermion anomalous dimensions:

$$
\begin{aligned}
\eta_{\phi}= & 4 v_{4} \kappa \lambda_{\phi}^{2} m_{2,2}^{4}\left(\frac{\nu}{2 \sqrt{\kappa}}, \frac{\nu}{2 \sqrt{\kappa}}+2 \kappa \lambda_{\phi} ; \eta_{\phi}\right) \\
& +4 N_{\mathrm{c}} v_{4} h^{2}\left[m_{4}^{(\mathrm{F}), 4}\left(\kappa h^{2} ; \eta_{\psi}\right)+\kappa h^{2} m_{2}^{(\mathrm{F}), 4}\left(\kappa h^{2} ; \eta_{\psi}\right)\right],
\end{aligned}
$$

$$
\begin{aligned}
\eta_{\psi}= & 2 C_{2}\left(N_{\mathrm{c}}\right) v_{4} g^{2}\left[(3-\xi) m_{1,2}^{(\mathrm{FB}), 4}\left(\kappa h^{2}, 0 ; \eta_{\psi}, \eta_{\mathrm{F}}\right)\right. \\
& \left.-3(1-\xi) \tilde{m}_{1,1}^{(\mathrm{FB}), 4}\left(\kappa h^{2}, 0 ; \eta_{\psi}, \eta_{\mathrm{F}}\right)\right] \\
& +v_{4} h^{2}\left[m_{1,2}^{(\mathrm{FB}), 4}\left(\kappa h^{2}, \frac{\nu}{2 \sqrt{\kappa}}+2 \kappa \lambda_{\phi} ; \eta_{\psi}, \eta_{\phi}\right)\right. \\
& \left.+m_{1,2}^{(\mathrm{FB}), 4}\left(\kappa h^{2}, \frac{\nu}{2 \sqrt{\kappa}} ; \eta_{\psi}, \eta_{\phi}\right)\right] .
\end{aligned}
$$

Including the appropriately adjusted fermion-boson translation as outlined in Sec. V, the flow equations for the minimum of the scalar potential and the scalar self-interaction read 


$$
\begin{aligned}
& \partial_{t} \kappa=-\left(2+\eta_{\phi}\right) \kappa+2 v_{4} \frac{\lambda_{\phi}}{\lambda_{\phi}+\frac{\nu}{4 \kappa^{3 / 2}}}\left[l_{1}^{4}\left(\frac{\nu}{2 \sqrt{\kappa}} ; \eta_{\phi}\right)\right. \\
& \left.+3 l_{1}^{4}\left(\frac{\nu}{2 \sqrt{\kappa}}+2 \kappa \lambda_{\phi} ; \eta_{\phi}\right)\right]-8 N_{\mathrm{c}} v_{4} h^{4} l_{1}^{(\mathrm{F}), 4}\left(\kappa h^{2} ; \eta_{\psi}\right) \\
& +\frac{2\left(\kappa \lambda_{\phi}-\frac{\nu}{2 \sqrt{\kappa}}\right)}{\left(\lambda_{\phi}+\frac{\nu}{4 \kappa^{3 / 2}}\right) h^{2}}\left(1-\kappa \lambda_{\phi}+\frac{\nu}{2 \sqrt{\kappa}}\right) \\
& \times\left[1+\left(1-\kappa \lambda_{\phi}+\frac{\nu}{2 \sqrt{\kappa}}\right) Q_{\sigma}\right]\left(\beta_{\lambda_{\sigma}}^{g^{4}} g^{4}+\beta_{\bar{\lambda}_{\sigma}}^{h^{4}} h^{4}\right), \quad \text { (C3) } \\
& \partial_{t} \lambda_{\phi}=2 \eta_{\phi} \lambda_{\phi}+2 v_{4} \lambda_{\phi}^{2}\left[l_{2}^{4}\left(\frac{\nu}{2 \sqrt{\kappa}} ; \eta_{\phi}\right)\right. \\
& \left.+9 l_{2}^{4}\left(\frac{\nu}{2 \sqrt{\kappa}}+2 \kappa \lambda_{\phi} ; \eta_{\phi}\right)\right]-8 N_{\mathrm{c}} v_{4} h^{4} l_{2}^{(\mathrm{F}), 4}\left(\kappa h^{2} ; \eta_{\psi}\right) \\
& +\frac{4 \lambda_{\phi}}{h^{2}}\left[1-2 \kappa \lambda_{\phi}+\frac{\nu}{\sqrt{\kappa}}+\left(1-\kappa \lambda_{\phi}+\frac{\nu}{2 \sqrt{\kappa}}\right)^{2} Q_{\sigma}\right] \\
& \times\left(\beta_{\lambda_{\sigma}}^{g^{4}} g^{4}+\beta_{\bar{\lambda}_{\sigma}}^{h^{4}} h^{4}\right) \\
& +\frac{16 \pi^{2} \lambda_{\phi}}{\nu h}\left(\frac{\nu}{2 \sqrt{\kappa}}-\kappa \lambda_{\phi}\right) d_{0}^{N_{\mathrm{c}}}\left(f_{\mathrm{c}} / k\right) C_{\mathrm{E}}\left(N_{\mathrm{c}}\right) \\
& \times\left(\frac{\alpha\left(k / f_{\mathrm{c}}\right)}{\alpha(\bar{\mu})}\right)^{-4 / b_{0}} \frac{1}{f_{\mathrm{c}}}\left(1+\frac{\left(-f_{\mathrm{c}}^{\prime}\right)}{f_{\mathrm{c}}} \partial_{t}\left(\kappa h^{2}\right)\right) .
\end{aligned}
$$

The Yukawa coupling flows in the broken regime according to

$$
\begin{aligned}
\partial_{t} h^{2}= & \left(2 \eta_{\psi}+\eta_{\phi}\right) h^{2}-4 v_{4} h^{4}\left[l_{1,1}^{(\mathrm{FB}), 4}\left(\kappa h^{2}, \frac{\nu}{2 \sqrt{\kappa}} ; \eta_{\psi}, \eta_{\phi}\right)\right. \\
& \left.-l_{1,1}^{(\mathrm{FB}), 4}\left(\kappa h^{2}, \frac{\nu}{2 \sqrt{\kappa}}+2 \kappa \lambda_{\phi} ; \eta_{\psi}, \eta_{\phi}\right)\right] \\
& -8(3+\xi) C_{2}\left(N_{\mathrm{c}}\right) v_{4} g^{2} h^{2} l_{1,1}^{(\mathrm{FB}), 4}\left(\kappa h^{2}, 0 ; \eta_{\psi}, \eta_{\mathrm{F}}\right), \\
& +2\left[1-2 \kappa \lambda_{\phi}-\frac{\nu}{\sqrt{\kappa}}+\left(1-\kappa \lambda_{\phi}+\frac{\nu}{2 \sqrt{\kappa}}\right)^{2} Q_{\sigma}\right] \\
& \times\left(\beta_{\bar{\lambda}_{\sigma}}^{g^{4}} g^{4}+\beta_{\bar{\lambda}_{\sigma}}^{h^{4}} h^{4}\right),
\end{aligned}
$$

and the flow of the axial anomaly is given by

$$
\begin{aligned}
\partial_{t} \nu= & -\left(3-\frac{\nu_{\phi}}{2}\right)+4 \pi^{2} \frac{\kappa \lambda_{\phi}-\frac{\nu}{2 \sqrt{\kappa}}}{h} d_{0}^{N_{\mathrm{c}}}\left(f_{\mathrm{c}} / k\right) C_{\mathrm{E}}\left(N_{\mathrm{c}}\right) \\
& \times\left(\frac{\alpha\left(k / f_{\mathrm{c}}\right)}{\alpha(\bar{\mu})}\right)^{-4 / b_{0}} \frac{1}{f_{\mathrm{c}}}\left(1+\frac{\left(-f_{\mathrm{c}}^{\prime}\right)}{f_{\mathrm{c}}} \partial_{t}\left(\kappa h^{2}\right)\right) \\
& +\frac{\nu}{h^{2}}\left[1+\left(1-\kappa \lambda_{\phi}+\frac{\nu}{2 \sqrt{\kappa}}\right)^{2} Q_{\sigma}\right]\left(\beta_{\bar{\lambda}_{\sigma}}^{g^{4}} g^{4}+\beta_{\bar{\lambda}_{\sigma}}^{h^{4}} h^{4}\right) .
\end{aligned}
$$

In these equations, the quantity $\beta_{\bar{\lambda}_{\sigma}}^{h^{4}}$ is also modified,

$$
\beta_{\lambda_{\sigma}}^{h^{4}}:=\frac{2}{N_{\mathrm{c}}} v_{4} \widetilde{l}_{1,1,1}^{\mathrm{FBB}), 4}\left(\kappa h^{2}, \frac{\nu}{2 \sqrt{\kappa}}, \frac{\nu}{2 \sqrt{\kappa}}+2 \kappa \lambda_{\phi} ; \eta_{\psi}, \eta_{\phi}\right),
$$

whereas $\beta_{\bar{\lambda}_{\sigma}}^{g^{4}}$ remains the same.
[1] C. Wetterich, Phys. Lett. B 301, 90 (1993); Nucl. Phys. B352, 529 (1991); Z. Phys. C 48, 693 (1990).

[2] H. Gies and C. Wetterich, Phys. Rev. D 65, 065001 (2002).

[3] Y. Nambu and G. Jona-Lasinio, Phys. Rev. 122, 345 (1961); 124, 246 (1961).

[4] J. Berges, N. Tetradis, and C. Wetterich, Phys. Rep. 363, 223 (2002).

[5] L.F. Abbott, Nucl. Phys. B185, 189 (1981).

[6] M. Reuter and C. Wetterich, Nucl. Phys. B417, 181 (1994); Phys. Rev. D 56, 7893 (1997); F. Freire, D.F. Litim, and J.M. Pawlowski, Phys. Lett. B 495, 256 (2000).

[7] M. Bonini, M. D’Attanasio, and G. Marchesini, Nucl. Phys. B421, 429 (1994); U. Ellwanger, Phys. Lett. B 335, 364 (1994).

[8] J.M. Pawlowski, Int. J. Mod. Phys. A 16, 2105 (2001).

[9] D.F. Litim and J.M. Pawlowski, J. High Energy Phys. 09, 049 (2002).
[10] J. Jaeckel and C. Wetterich, Phys. Rev. D 68, 025020 (2003).

[11] D.U. Jungnickel and C. Wetterich, Phys. Rev. D 53, 5142 (1996).

[12] R.S. Chivukula, M. Golden, and E.H. Simmons, Phys. Rev. Lett. 70, 1587 (1993).

[13] F. Hoefling, C. Nowak, and C. Wetterich, Phys. Rev. B 66, 205111 (2002).

[14] K.I. Aoki, K.I. Morikawa, J.I. Sumi, H. Terao, and M. Tomoyose, Prog. Theor. Phys. 97, 479 (1997).

[15] K.I. Aoki, K. Takagi, H. Terao, and M. Tomoyose, Prog. Theor. Phys. 103, 815 (2000).

[16] D.F. Litim, Phys. Lett. B 486, 92 (2000); Phys. Rev. D 64, 105007 (2001).

[17] U. Ellwanger, M. Hirsch, and A. Weber, Z. Phys. C 69, 687 (1996); Eur. Phys. J. C 1, 563 (1998).

[18] D.F. Litim and J.M. Pawlowski, Phys. Lett. B 435, 181 (1998). 
[19] G. 't Hooft, Phys. Rev. D 14, 3432 (1976); 18, 2199(E) (1978); M.A. Shifman, A.I. Vainshtein, and V.I. Zakharov, Nucl. Phys. B163, 46 (1980); E.V. Shuryak, ibid. B203, 93 (1982).

[20] For recent reviews, see T. Schafer and E.V. Shuryak, Rev. Mod. Phys. 70, 323 (1998); D. Diakonov, Prog. Part. Nucl. Phys. 51, 173 (2003).

[21] J.M. Pawlowski, Phys. Rev. D 58, 045011 (1998).

[22] K.I. Kondo, M. Tanabashi, and K. Yamawaki, Prog. Theor. Phys. 89, 1249 (1993); K.I. Kubota and H. Terao, ibid. 102, 1163 (1999); M. Reenders, Phys. Rev. D 62, 025001 (2000).

[23] C. Wetterich, Phys. Lett. B 209, 59 (1988); S. Bornholdt and C. Wetterich, ibid. 282, 399 (1992).

[24] E. Eichten et al., Phys. Rev. Lett. 34, 369 (1975); 36, 1276(E) (1976); T. Barnes, F.E. Close, and S. Monaghan, Nucl. Phys. B198, 380 (1982); S. Godfrey and N. Isgur, Phys. Rev. D 32,
189 (1985); A.C. Mattingly and P.M. Stevenson, Phys. Rev. Lett. 69, 1320 (1992); A.C. Aguilar, A. Mihara, and A.A. Natale, hep-ph/0208095.

[25] L. von Smekal, R. Alkofer, and A. Hauck, Phys. Rev. Lett. 79, 3591 (1997); Ann. Phys. (N.Y.) 267, 1 (1998); 269, 182(E) (1998); D. Atkinson and J.C. Bloch, Phys. Rev. D 58, 094036 (1998); D. Zwanziger, ibid. 65, 094039 (2002); C. Lerche and L. von Smekal, ibid. 65, 125006 (2002).

[26] F.D. Bonnet, P.O. Bowman, D.B. Leinweber, A.G. Williams, and J.M. Zanotti, Phys. Rev. D 64, 034501 (2001); J.R. Bloch, A. Cucchieri, K. Langfeld, and T. Mendes, hep-lat/0209040.

[27] C.S. Fischer and R. Alkofer, Phys. Lett. B 536, 177 (2002).

[28] D.V. Shirkov and I.L. Solovtsov, Phys. Rev. Lett. 79, 1209 (1997); Teor. Mat. Fiz. 120, 482 (1999) [Theor. Math. Phys. 120, 1220 (1999)].

[29] H. Gies, Phys. Rev. D 66, 025006 (2002). 